\title{
Serotonin and sensorimotor adaptation in Drosophila larvae
}

\author{
Mary Catherine Farrell \\ Wilmington, $\mathrm{OH}$ \\ B.A., Biology, Hiram College 2011 \\ Hiram, $\mathrm{OH}$
}

A thesis presented to the faculty of the University of Virginia for the completion of a Doctor of Philosophy

Department of Biology

University of Virginia

December, 2015 
Abstract

Locomotion requires specialized neural circuits called central pattern generators (CPGs) that tightly control the movement of specific body parts. These circuits need to control crawling in a potentially changing environment, and in the presence of potential injuries. How these CPGs respond to localized disruptions is not well understood. To address this, we attached a splint to two abdominal segments on the right side of the larva, and observed their crawling behavior. The splint initially causes an exaggerated side-to-side movement in the posterior, characterized by a difference between the left and the right of segment A5 compressing. After four hours, the side-to-side movement is reduced and the left and right of A5 compress at the same time. Larvae are able to deadapt over a very short period of time after the splint was removed. These results indicate that changes in segment connections are capable of occurring in response to different stressors.

Adaptation occurs more rapidly in the presence of increased serotonin, and fails to occur when serotonin levels have decreased. Serotonin's action in adaptation is dependent upon signaling through the 5-HT7 receptor expressed specifically in glial cells. In addition, we describe an assay that can be used to understand neuromodulators, genetics, and anatomical components of plasticity and change in CPGs and sensorimotor circuits. In an unexpected result, we discovered a temperature-sensitive component to adaptation. Larvae exposed to higher temperature have a reduced asymmetric posterior movement, which returns when larvae are retuned to room temperature. 


\section{Acknowledgements}

I owe a large debt of gratitude to my advisor and committee. Dr. Barry Condron, for giving me the opportunity to work on a project that I found fascinating, and the chance to work with some of the most remarkable undergraduates at the University of Virginia. Dr. Iggy Provencio, for being an incredible mentor, for always being available, and for providing wonderful feedback during my qualifier all the way until now. Dr. Sarah Kucenas, for providing more encouragement and more of her time than I could have ever asked for. Thank you for being a wonderful mentor, with grad school matters as well as personal ones. Dr. Adrian Halme, for asking the hard questions, and for providing me with tools and suggestions that I have proven to be invaluable to the development and completion of my project. Thank you so much for making the trek over to PLSB for each of my committee meetings. And finally Dr. Sarah Siegrist, for letting me bounce ideas off of you about my project before you even moved to Charlottesville. Having you and your lab members in such close proximity made all of the difference in the world.

To Cara Constance and Nick Hirsch, thank you for making my undergraduate experience one where I grew to love neuroscience and was first able to experience the excitement of conducting my own research.

To Erin Kodis, Brittany Sutherland, Catherine Debban, Matt Pahl, Rich Smindak, Marisa Adhikusuma, Robbie and Patricia Hott for being wonderful friends. All of my 
fondest memories of the last 4 years involve some combination of you folks, and they all make me smile.

To Brittany Brunner, Rachael Armstrong, and Lauren Strider for being my favorite old friends, and providing mass quantities of free counseling, advice, and laughter.

My grandparents - David, Maxine, Zo, John, and Lou. I will never forgl of the lessons in plant identification, crocheting, cooking and gardening I learned from you, Grandma Max. I will always cherish the medical terminology, large vocabulary words, and appreciation for brownie batter that I learned while sitting on the couch with you, Grandpa Dave. I will always strive for your unconditional warmth and the lessons you taught me about family, Grandpa John. And Grammy Zo and Lou - thank you for teaching me how to laugh, especially at myself.

Aunt Cathy and Uncle Jim - thank you. It has taken me a while to understand just how lucky I am to have an Aunt and Uncle who have been there to the extent that you two have. Thank you for never missing a graduation, or special occasion, and for always making it to my birthday, regardless of the snowfall.

To my wonderful family - Tom, Jonda and John for everything. I would not be where I am today had I not been a part of such a loving, nurturing, and accepting family. 
Finally, my wonderful husband Zack. For making the last two years more fun and more fulfilling than I could have ever hoped for. 
I. An introduction to Central Pattern Generators and their regulation Chapter 2 - Materials and Methods

I. Fly strains

II. Sensorimotor plasticity assay 30

III. Data analysis and quantification 31

IV. Pharmacological treatments 32

V. Temperature shifts 33

Chapter 3 - The larval sensorimotor circuit exhibits plasticity in response to partial immobilization

I. Abstract

II. Introduction

III. Results

a. Immobilizing individual segments causes a unique crawling pattern, which is corrected after 4 hours

b. Asymmetric segment compression results in errors in peristaltic wave propagation 
c. Immobilized segments are compensated for with changes in surrounding body segments

IV. Conclusion

I. Abstract

II. Introduction

III. Results

63

a. Increasing serotonin levels in larvae increases the rate of sensorimotor adaptation

b. Decreasing serotonin pharmacologically prevents adaptation from occurring

c. Elevated serotonin transporter causes very rapid adaptation 66

d. An RNAi-based screen revealed that expression of the 5-HT7 receptor on glial cells is crucial for adaptation.

e. Additional results from the RNAi screen

IV. Discussion

II. Introduction

III. Results 
a. Asymmetric phenotype is reversed at higher temperature 81

$\begin{array}{ll}\text { IV. Discussion } & 83\end{array}$

Chapter 6-General Discussion 86

$\begin{array}{ll}\text { I. Summary } & 87\end{array}$

II. Serotonin regulates adaptation in the larval sensorimotor circuit 89

III. The 5-HT7 receptor could potentially have a multifaceted role in $\begin{array}{ll}\text { adaptation } & 91\end{array}$

IV. Glia are crucial regulators of sensorimotor plasticity 92

V. Temperature influences adaptation, either through sensory neurons or by $\begin{array}{ll}\text { altering substrate properties } & 94\end{array}$

VI. Future Directions 95

VII. What are the connections between CPGs? 97

VIII. Similarities between turning and asymmetric crawling 100

IX. Beyond Flies: What can be learned from other model systems 101

$\begin{array}{lr}\text { Appendix } & 102\end{array}$

$\begin{array}{ll}\text { Works Cited } & 106\end{array}$ 


\section{Table of Figures}

Table 1 - A partial list of sensory and interneurons important for locomotion and locomotion-related phenotypes in Drosophila larvae

Fig. 1.1 - Central Pattern Generators control several rhythmic behaviors

Fig. 1.2 - CPGs in the ventral nerve cord govern locomotion

Fig. 2.1 - Schematic of analysis for larval locomotion

Fig. 2.2 - Schematic of the temperature shift scheme used to address the role of temperature in adaptation

Fig. 3.1 - Larvae crawl using a series of interconnected CPGs, each controlling the left or right of a specific abdominal segment.

Fig. 3.2 - Larvae adapt in a sensorimotor plasticity assay

Fig. 3.3 - Splinting the right of A6 and A7 results in asynchrony between the left and the right of anterior segments 
Fig. 3.4 - Compression of the left side of A5 is required for peristaltic wave propagation in the presence of the original splint.

Fig. 3.5 - After plastic removal, the pattern of segment compression reverts to the pattern observed in wild type larvae.

Fig. 3.6 - Compression of segment A6 is not required for compression in larvae that have already adapted.

Supplemental Figure S3.1 - Canton S larvae respond differently to the presence of a splint than either Oregon R or SN/GFP

Fig. 4.1 - Increasing serotonin results in rapid adaptation of the sensorimotor system.

Fig. 4.2 - Decreasing serotonin synthesis prevents adaptation by 240 minutes.

Table 4.1 - An RNAi-based screen revealed a dependence on the 5-HT7 receptor in glial cells for normal sensorimotor adaptation.

Fig. 4.3 - Overexpression of the serotonergic transporter in serotonergic neurons results in very rapid adaptation. 
Fig. 4.4 - Glial specific knockdown of the 5-HT7 receptor prevents larvae from successfully adapting 240 minutes after adaptation.

Fig. 5.1 - The asymmetric posterior movement transiently disappears at elevated temperatures. 
Chapter 1: An introduction to central pattern generators and their regulation 
Organisms rely on circuits within their nervous system to carry out many of the functions crucial for survival. Using very simple components, our nervous system is able to generate many robust and predictable behaviors which govern our day to day lives. Relatively simple feedback and regulation allow small groups of neurons to generate patterns of activity to carry out very important rhythmic functions. Respiration, locomotion, and peristaltic gastrointestinal movements all rely on neural circuits acting predictably in a rhythmic fashion. Underlying each of these processes are specialized neural circuits called central pattern generators (CPGs), which ensure rhythmicity and maintain a specific pattern of motor output (Friesen, 1994).

Although distinct CPGs govern each of these behaviors, CPGs in both vertebrates and invertebrates are made up of common cellular components; interneurons, where the circuit's rhythmic activity is generated, motor neurons which signal to the crucial muscle groups and carry out the circuit's specific activity, and sensory neurons which provide the circuit with regulatory feedback (Friesen, 1994).

Interneurons found in the CNS are responsible for generating the rhythmic activity of the circuit (Friesen, 1994). The nature of these interneurons vary with the specific circuit, but in general they consist of both excitatory and inhibitory cellular components. In the simplest form, inhibitory neurons reciprocally inhibit each other, while receiving chronic excitatory input resulting in an alternating "on- 
off' pattern as the output (Friesen, 1994). Perhaps the best studied example of interneurons in a CPG controlling motor output is in the crab (Cancer borealis) stomatogastric ganglion: a group of neurons which controls digestion. The core pacemaker is primarily made up of three interneurons, called MCN1, LG and Int1. MCN1 utilizes GABA and the cotransmitter CabTRP to regulate the excitability of LG and Int1, which in turn alternatively inhibit one another. Several other neurons and modulators feed into this circuit and trigger initial activity, but these neurons provide the driving force for gastric milling during digestion (reviewed in Marder and Bucher, 2001 and Blitz and Nusbaum, 2012). Virtually every neuron in this CPG has been characterized, as well as how many of the individual neuromodulators contribute to the function of the entire network. However, CPGs in higher organisms tend to be more complicated, and much remains to be understood about their regulation.

Motor neurons downstream of the interneurons are responsible for controlling the movement of the muscles involved in the output of that particular CPG. Motor neuron cell bodies are found in the central nervous system (CNS), and form synapses with the interneurons generating the pattern of activity. In addition to regulation from the CPG, the excitability of motor neurons can also be modified as a means to regulate their function (Burke, 2001). Changing the excitability of a given neuron alters the likelihood that it will fire in response to a controlled stimulus, thereby changing when or how it may fire. In the cardiac ganglion of crab, for example, the excitability of neurons in the CPG can be regulated by the expression of 
the genes which encode the channels responsible for the $\mathrm{I}_{\mathrm{A}}$ and $\mathrm{I}_{\mathrm{KCa}}$ currents (Ransdell, 2012).

The specific muscles controlled by motor neurons vary based on what function is carried out by the CPG. For example, during locomotion, motor neurons control groups of muscles in either the limbs or the body segments which contribute to directional movement. During respiration, the diaphragm and intercostal muscles are active in order to control lung expansion. During digestion, motor neurons control the progression of food through the intestines. It can be difficult to visualize, but at the anatomical level these muscle groups also exist in repeating units (Fig. 1.1).
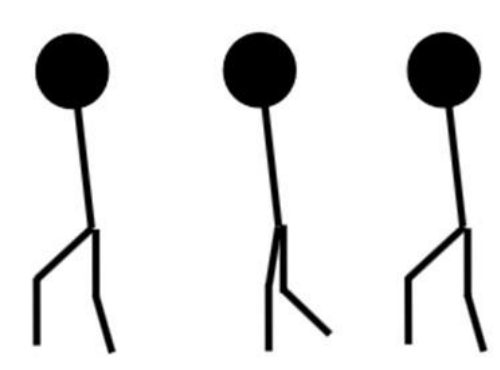

Locomotion

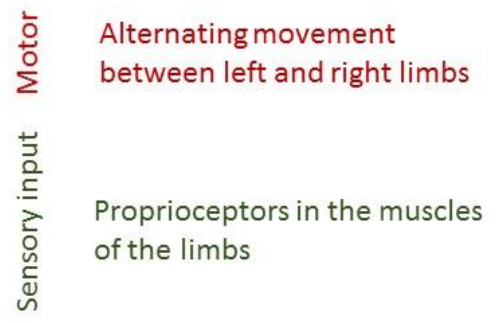

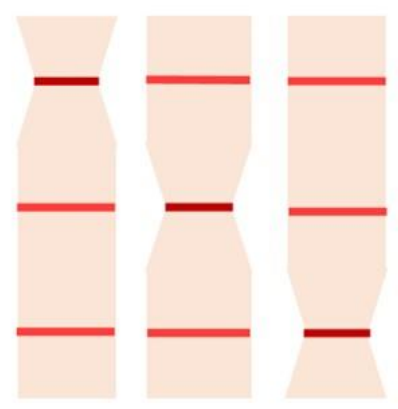

Gut Peristalsis

Sequentially compressing muscles in the intestine

Mechanoreceptors in the gut responding to the presence of food

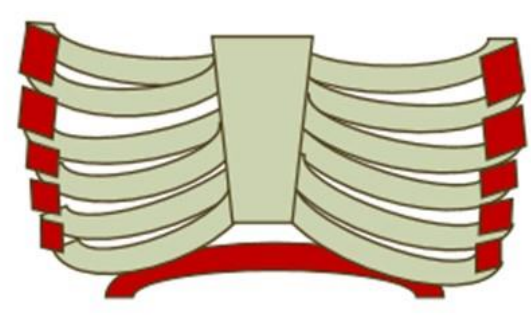

\section{Respiration}

Inhalation and exhalation is controlled
compressing and relaxing the
intercostal muscles and diaphragm
Chemosensory neurons in the CNS
respond to $\mathrm{CO}_{2}$ and pH levels in the
bloodstream

Fig. 1.1 - CPGs govern several rhythmic behaviors in the body, including locomotion, gut peristalsis, and respiration. The CPG responsible for controlling locomotion generates alternating movement between the left and the right of each limb, and 
utilizes positional information from proprioceptors to modify their output. Gut peristalsis is carried out by sequential constriction of muscle segments in the intestines. Mechanical stress from the presence of a food mass indicates where the food is moving. Respiration utilizes intercostal and diaphragm muscles to cause air to flow in and out of the lungs. Chemosensory neurons in the brain can cause an increase in the rate of respiration in response to increased $\mathrm{CO}_{2}$ and decreased $\mathrm{pH}$.

Locomotion and digestion are examples of rhythmic behaviors that are carried out by coordinating several body parts and muscle groups. As a result, CPGs are often composed of smaller, interconnected CPGs that specifically modulate individual muscle groups. For example, in the Drosophila larval ventral nerve cord (VNC), interneurons exist in repeating units corresponding to each of the larval body segments, with paired left and right segments. Each section contains a group of identical interneurons and motor neurons in the VNC (Heckscher, 2012). This organization allows for the muscles in individual body segments to act as units, functioning in response to activity in neighboring segments (reviewed in Butt and Keihn, 2003).

CPGs coordinate movement between limbs and body segments

The CPGs controlling locomotion are unique in that the often need to coordinate activity between multiple limbs and body segments in order to ensure effective locomotion. In many cases, this requires the work of multiple CPGs, each controlling a specific segment or limb working together to accomplish movement. These CPGs are connected, and are thought to influence the activity of neighboring 
segments. In mammals, a group of commissural interneurons which express the homeobox gene $D b x 1$ generate are responsible for coordinating left and right limb movement, by inducing a time delay between the left and right limb movement (Lanuza, 2004). This communication is crucial for coordinating movement between abdominal segments to generate efficient locomotion. In $D b x 1$ mutants identified by Lanuza and colleagues, mice had an increased tendency to move both hind limbs at the same time, rather than alternating their movement as wild-types do (Lanuza, 2004).

Similarly to mammals, leeches swim by asymmetrically compressing individual sections of their abdominal muscles (Stent, 1978). In other words, when the left side is activated, or compressed, the right side is relaxed (Stent, 1978). Organisms such as insect larvae move by simultaneously moving the left and right of individual body segments (Heckscher, 2012). As a result, coordination is maintained through slightly different cellular machinery. Recently, a group of interneurons expressing the hox gene Evenskipped have been identified as an evolutionarily conserved cell type which is crucial for maintaining symmetric compression (Heckscher, 2015). The fact that these mechanisms are so widely conserved speaks to the importance of coordination between CPGs. However, very few studies address the role of prolonged disrupted coordination in CPG networks. Sensory input modifies CPG output

Although oscillation of CPGs can occur without any additional input, sensory neurons provide these circuits with information about the specific activity that they are generating. For example, the CPG which controls respiration receives input from 
chemosensory neurons in the brain, which detect changing levels of carbon dioxide, and this CPG can cause an increased rate of respiration, as well as a fear response. Defects in the activity of these chemosensory neurons have been linked to cases of sudden infant death syndrome (SIDS) in humans (Weese-Mayer, 2003). In these cases, increased levels of $\mathrm{CO}_{2}$ would normally arouse breathing. However, it is thought that in SIDS cases, the arousal signals are not activated in response to their chemical signals (Buchanan, 2010).

Locomotion utilizes sensory input from mechanosensitive neurons to control the rate of motor activity. Leech (Cang and Friesen, 2002), Drosophila (Song, 2007), tobacco hornworm (Manduca) (Simon, 2009) all require input from peripheral sensory neurons in the body wall in order to regulate the oscillation of the interneurons in the CPG. In Manduca, activity in the sensory neuron SRO feeds into the CPGs which govern locomotion (Simon, 2009). Similar neurons Drosophila larvae are required for locomotion (Song, 2007) and provide direct input to the interneurons required for symmetric movement (Heckscher, 2015). In leech, the sensory neurons have been shown to directly influence the rate of activity of the pattern generating neurons in the CNS (Cang, 2002). The importance of sensory input to CPGs is well documented across many species. However, it is not currently understood how symmetry is maintained or how networks of CPGs respond to aberrant sensory input.

In leech, Manduca, and Drosophila larvae individual body segments possess a set of nearly identical sensory neurons which provide feedback to the CPG. However, it is not currently well understood how the sensory input of an individual 
segment contributes to the activity of surrounding body segments. Fox and Wu conducted a series of experiments in which they severed the nerve bundles (containing sensory and motor neurons) to individual segments. When they did, they observed abnormal timing and degree of compression of the surrounding abdominal segments, including those anterior and contralateral to the segment where the nerves were cut (Fox and $\mathrm{Wu}, 2006$ ). When multidendritic (MD) neurons, a class of mechanosensitive neurons which contain several proprioceptors, were silenced, larvae eventually became totally immobilized (Song, 2007). Within that class, no individual group of neurons is required for forward crawling, but several groups of sensory neurons influence different aspects of forward locomotion (Hughes and Thomas, 2007). Table 1.1 shows a partial list of sensory neurons and interneurons which are known to regulate locomotion of Drosophila larvae. The current literature model of how sensory neurons influence interneurons and motor output is shown in figure 1.2. 

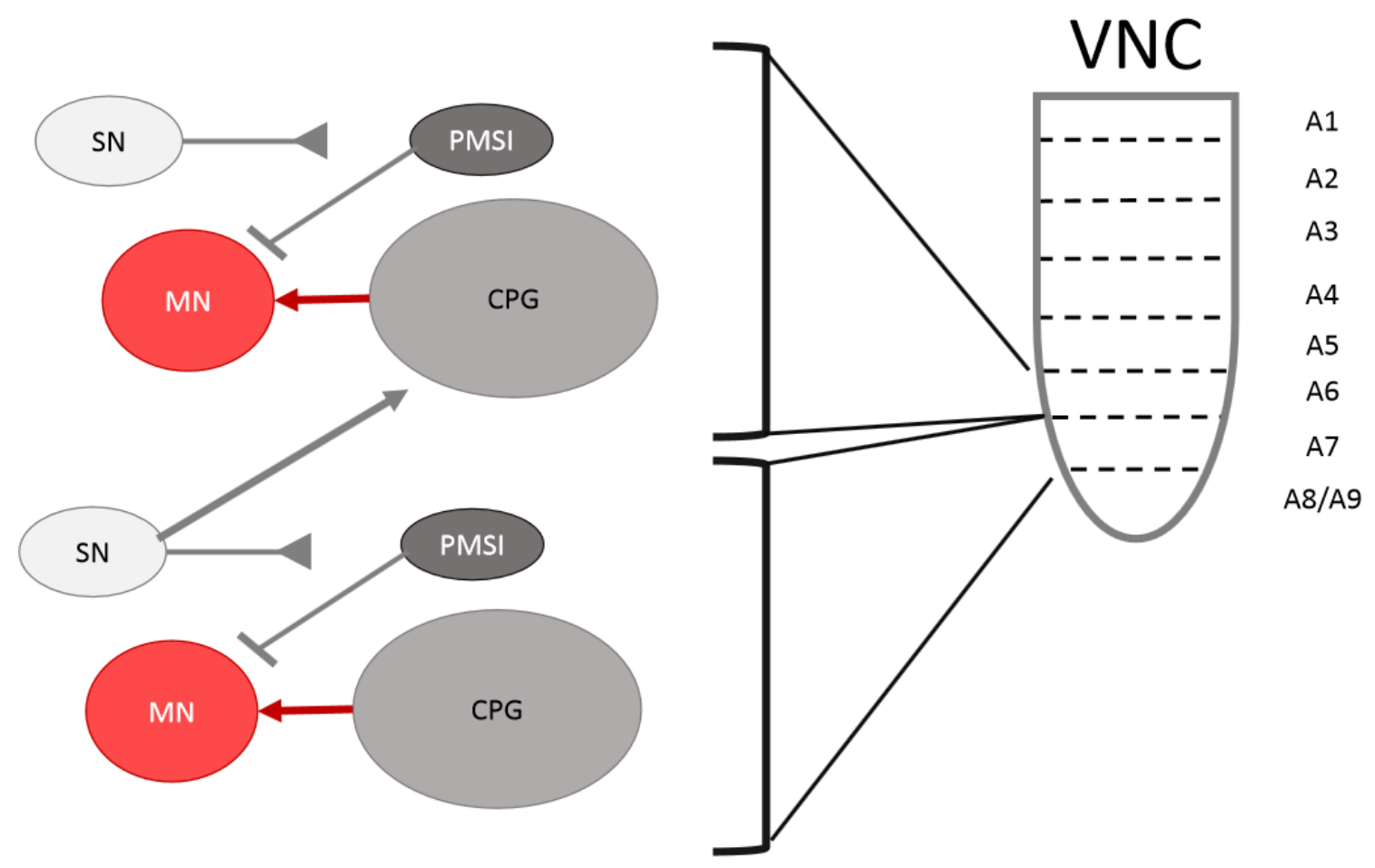

Fig. 1.2 - Our current understanding of the neural circuitry controlling larval crawling. CPGs, made up of several groups of interneurons, cause the motor neurons to fire in a specific segment. After the motor neuron fires, it is inhibited by period-expressing medial segmental interneurons (PMSI, dark grey). The sensory neurons (light grey), specifically the multidendritic (MD) sensory neurons, fire when the segment compresses, contributing to the compression of the anterior segment. This model is based on work by Song, 2007, and Kohsaka, 2014. It is unclear what post-synaptic partners of the sensory neurons are. Lines indicate connections between neurons or groups of neurons. Lines that terminate with arrows indicate an excitatory connection, lines that terminate with lines indicate inhibition, and lines that end in triangles are used to indicate connections which are not as well understood. 


\begin{tabular}{|c|c|c|}
\hline Neuron & Phenotype & Resource \\
\hline \multicolumn{3}{|l|}{ Sensory } \\
\hline Multidendritic neurons & Silencing with ShibireTS arrests locomotion & Song, 2007 \\
\hline $\begin{array}{l}\text { Bipolar Dendritic (bd) } \\
\text { neurons }\end{array}$ & Cell-specific knockdown of nompC significantly decreases speed of locomotion & Cheng, 2010 \\
\hline \multirow[t]{2}{*}{ Chordotonal organs (cho) } & Mutations in cho neuron development results in abberant turning & Caldwell , 2003 \\
\hline & Expression of TRPV1 required in cho for hearing & Kim 2003 \\
\hline Class I MD Neurons & Cell-specific knockdown of nompC significantly decreases speed of locomotion & Cheng, 2010 \\
\hline Class III MD neurons & Express rippedpocket and nompC to elicit responses to light touch & Tsubouchi , 2012 \\
\hline \multirow[t]{2}{*}{ Class IV MD neurons } & Expression of painless in MD neurons required for nocicpetion & Tracey, 2003 \\
\hline & Activation with ChR shows nocefensive rolling phenotype & Hwang , 2007 \\
\hline \multicolumn{3}{|l|}{ Interneurons } \\
\hline Serotonergic & Mediates turning behavior & Okusawa , 2014 \\
\hline Leucokinin-exressing & Mediates turning behavior & Okusawa , 2014 \\
\hline $\begin{array}{l}\text { Period-positive Medial } \\
\text { Segmental Interneurons }\end{array}$ & Directly govern the activity of the interneurons during crawling & Kohsaka , 2014 \\
\hline
\end{tabular}

Table 1 - A partial list of sensory and interneurons involved in locomotion.

Similarly, in Manduca, the SRO neuron provides excitatory input to the segments' anterior and contralateral motor neurons (Tamarkin and Levine, 1996). All of these studies have been conducted by looking in "semi-intact preparations". Larvae prepped in this way are dissected such that the CNS and nerve bundles are intact, but the larva is immobilized in a saline solution, allowing the body wall muscles to compress in a way that creates "fictive locomotion" (Pulver, 2015). While these systems are incredibly powerful and provide insight into the electrophysiological properties of CPGs, experiments conducted with this preparation can only be conducted over a very short period of time. Using HL3 dissection solution, larvae have been observed to contract for tens of minutes (Fox and $\mathrm{Wu}, 2006)$, but larvae have not been reported to compress at any later time 
points. This makes it impossible to tell how larvae would respond to prolonged periods of time with disrupted sensory input. It is currently not understood how longer periods of sensory disruption influence CPGs, nor does there exist a good way to experimentally address this question.

The impact of neuromodulators: Serotonin and Tyramine

Semi-intact preparations are one of the best ways to parse out the impact of neuromodulators or other signaling components in circuit function. One of the great strengths of this application is that the muscles and nervous system can be simply added to the dissection solution as the larvae are being observed. For example, Dasari and Cooper have shown, using this preparation, that serotonin and dopamine influence the activity of motor neurons either directly, or in response to changes upstream in the CNS (Dasari and Cooper, 2004). Tyramine and octopamine, the Drosophila equivalents of epinephrine and norepinephrine, have also been shown to play a role in larval locomotion. Increased tyramine and decreased octopamine causes a drastic increase in pausing episodes, and a decrease in linear crawling (Saraswati, 2003). In addition, tyramine influences the ability of surrounding segments to maintain appropriate timing during fictive locomotion (Fox and $\mathrm{Wu}$, 2006). Octopamine is necessary for the initiation of a peristaltic wave by causing compression of the posterior-most segment (Fox and $\mathrm{Wu}, 2006)$.

Out of all of the neuromodulators studied in the context of larval locomotion, serotonin has been studied very thoroughly and has a large role in many subtle aspects of crawling. Serotonin and leucokinin-expressing neurons are required for proper turning forward crawling (Okusawa, 2014). Decreasing the expression of 
serotonin receptors in different parts of the mushroom bodies (MBs, analogous to the hippocampus) in the CNS have been found to either increase or decrease the rate of locomotion (Silva, 2014). Serotonergic neurons, by eliciting a response in leucokinin-expressing neurons, control turning behavior in crawling larvae (Okusawa, 2014). Although serotonin isn't required for larval crawling, serotonin plays an incredibly important role in many subtle aspects of larval locomotion.

Serotonin plays a large role in sensorimotor circuits in other organisms as well, both in their development and their function in mature organisms. Much of the early work establishing the importance of serotonin in motor function was originally conducted in mice, rat, lamprey and cat models. In isolated spinal cord preparations of rat (Cazalets, 1992), lamprey (Harris-Warrick, 1985), rats (FeraboliLohnherr, 1999), and cats (Barbeau, 1990). Zebrafish require normal serotonin levels in order for the proper development of their CPGs which govern swimming (Brustein, 2003). Across evolution, serotonin is incredibly important for regulating limb coordination to carry out efficient locomotion.

Serotonin coordinates other CPGs

In addition to being central to many aspects of locomotor CPGs, serotonin is crucial for regulating the activity of other CPGs as well. The role of serotonin in the respiratory CPGs has been especially well documented (Millhorn, 1980). Of great medical significance is the link between serotonin and sudden infant death syndrome (SIDS) and polymorphisms of the serotonin transporter (SERT) in chemosensory neurons in the brainstem. GWAS studies have identified a specific loss-of-function polymorphism found in SERT to an increased likelihood of death by 
SIDS (Weese-Mayer, 2003). In order to trigger arousal in response to increased $\mathrm{CO}_{2}$ levels, the chemosensory neurons in this circuit need to express functional SERT (Buchanan, 2010). In fact, treating newborn mice with fluoxetine (a commonly prescribed SSRI) diminishes their ability to respond to decreased blood $\mathrm{pH}$ (Voituron, 2010). It is important to point out that SERT isn't required for normal respiratory CPG function, but it is only required to elicit a physiological response to changes in $\mathrm{CO}_{2}$ levels in the blood. In other words, the serotonin is required in order for the circuit to adapt to altered sensory stimuli.

Serotonin is also known to influence another aspect of respiratory CPG plasticity. Normally, in response to hypoxic conditions, organisms increase the tidal volume of respiration, called the phrenic motor response (Baker, 2000). In studies conducted with anesthetized rats, inhibiting the expression of the 5-HT7 receptor has been shown to prevent the phrenic motor response when rats are shifted to a hypoxic environment (Hoffman, 2011). As was the case with serotonin's role in SIDS, the 5-HT7 receptor in this circuit is not required for its normal function. The 5HT7 receptor is only required when the circuit needs to adapt in response to a change in sensory input. In the case of SIDS, the circuit was unable to respond to an increase in $\mathrm{CO}_{2}$ and $\mathrm{pH}$, and in the case of the phrenic motor response the circuit is unable to respond to the hypoxic environment.

Another CPG which requires serotonin is in the CPG controlling gut peristalsis. As shown in figure x.x, muscles in the gut compress sequentially, to allow for food to move throughout the digestive tract. Interestingly, there is more serotonin present in the enteric nervous system than in the central nervous system, 
suggesting an incredibly important role for serotonin in the neuronal control of digestion (Gershon, 1991). In Drosophila, serotonergic neurons in the subesophageal ganglia are known to have a profound influence on the activity of motor neurons and pattern-generating interneurons which are responsible for processing food (Schoofs, 2014). It is thought that interstitial cell of Cajal (ICC) are the pacemaker cells which control the compression of the gut in a sequential fashion, although there are thought to be additional compensatory mechanisms (Huizinga and Lammers, 2009). Although most people are familiar with serotonin's role in disorders such as depression and anxiety, there are several disorders of the digestive system which are treated with drugs targeting the serotonergic system (Costedio, 2006). Amityptaline, a tricyclic antidepressant, is commonly prescribed for irritable bowel syndrome (IBS) (Rajagopalan, 1998) and cyclic vomiting disorder (Andersen, 1997), both disorders characterized by aberrant compression of the stomach and intestines.

The major CPGs in vertebrates and invertebrates require serotonin for their ability to function normally in specific conditions. What is especially fascinating, is that serotonin isn't necessarily required for normal CPG function in most situations, but it is often crucial for the ability of these circuits to respond to changes in response to sensory input. The fact that this is conserved across many different organisms and CPGs which control a wide variety of sensory functions invite us to speculate what other subtle roles serotonin is playing in these physiological functions. One question that remains unanswered in the literature, is how symmetric CPGs maintain symmetry, and respond to prolonged periods of induced 
asymmetry. We can point to Heckscher and colleagues, who have identified the neurons responsible for maintaining symmetry in Drosophila larvae, but those larvae were imaged over a very short period of time. While these insights are important, it is possible that another phenotype could be observed later in adaptation. In addition, to date no one has induced sensory asymmetry in a crawling larva and observed the impact on the overarching crawling behavior. However, with such a large amount of cellular machinery dedicated to maintaining symmetry between the two sides, it stands to reason that the larval sensorimotor CPGs would respond to prolonged periods of asymmetry. What is needed in the field is a way to observe how asymmetry is dealt with in crawling larvae.

We hypothesize that, if a small number of abdominal segments are forced to compress asymmetrically, the activity in the surrounding abdominal segments would change, due to the high connectivity between individual hemisegments. However, the importance of symmetric movement, we would not expect these abnormal patterns of activity to persist. To this end, this dissertation describes a sensorimotor plasticity assay, to induce asymmetry in the abdominal segments of crawling larvae. To induce the asymmetry, we attached a plastic splint to the right side of a small number of abdominal segments. We observed a unique writhing phenotype immediately after attaching the splint, characterized by excessive "swinging" of the posterior end during forward crawling. With this excessive posterior movement, we observed a significant discrepancy between when the left and the right of each abdominal segment. This asynchrony was most evident in the segments immediately anterior to the abdominal segments. After a period of 4 
hours, we observed the swinging phenotype diminish, and larvae were able to crawl normally. We observed that this behavior was reversible. Because CPGs are known to be regulated tightly by neuromodulators, we hypothesized that a neuromodulator controls the rate of adaptation. We hypothesized that this neuromodulator was very likely to be serotonin, because of its known role in mediating more subtle aspects of locomotion. Indeed, adaptation is mediated by serotonin, as we have demonstrated through pharmacological manipulation. Although it is unclear how serotonin mechanistically brings about adaptation, we learned through an RNAi screen that serotonin acts through the glial 5-HT7 receptor. Using these data, we propose a model describing the interaction of CPGs between segments, as well as some of the cellular and molecular signaling components which may be involved. In this dissertation I will discuss support for our model in the literature, and future experiments to address alternative hypotheses. 
Chapter 2 - Materials and Methods 
Fly strains

All wild-type experiments were conducted using Oregon R (OR) larvae unless otherwise stated. Flies were raised in standard conditions at $25^{\circ} \mathrm{C} .24$ hour egg collections were made on molasses agar plates (2.75\% agar, $11.25 \%$ molasses) with yeast paste. After approximately 92 hours, foraging $3^{\text {rd }}$ instar larvae were selected and moved to a fresh molasses agar plate for the duration of the assay. Oregon $\mathrm{R}$ (OR) (Bloomington Stock Center, Bloomington, IN) larvae were used in these experiments.

Sensorimotor plasticity assay

To perform the sensorimotor assay, foraging $3^{\text {rd }}$ instar larvae were used. The splints used were pieces of plastic that are $0.92(+/-0.02) \mathrm{mm} \times 0.63(+/-0.01) \mathrm{mm}$. Splints were attached with Gorilla glue (The Gorilla Glue Factory, Cincinnati, OH) to the right side of the larva on segments A6 and A7. After the splint was attached, the larvae were allowed to crawl freely around the plate for the duration of the assay. In experiments where plastic was removed, a dental pick was used to pry the splint off of the abdomen. Occasionally, we would observe darkened scarring where the plastic had been located, but the segment was still able to contract. We observed larvae crawling at each time points. We observed no difference in the distance larvae crawled at different phases during adaptation. Although these observations were qualitatively observed, they were never quantified. 
Up to three waves were recorded per larva per time point. Larvae were filmed using a Kodak Zi8 camera with a macro lens in 1080p resolution at 60 frames per second.

\section{Locomotion analysis}

Videos were analyzed using ImageJ. For general adaptation analysis, videos were first reduced from 60 to $20 \mathrm{~Hz}$. Using the manual tracker plugin, the movement of the posterior end of the larva was measured by following the movement of the spiracles during peristaltic crawling. The $\mathrm{x} / \mathrm{y}$ coordinates of the spiracle movement, the output of the manual tracker plugin, were graphed with the wave start and stop rotated to start and stop at $y=0$. The height of the peak was then obtained as a measure of oscillation height. For measuring segmental coordination, videos were not reduced in frame frequency. A segment was designated to start compressing in the first frame in which it was observed to shorten, and it was designated to stop compressing in the first frame in which it was observed to relax. All waves were registered, with the value of 0 being assigned to when the first segment begins to compress, and the value of 1 being assigned to when the last segment begins to compress. 

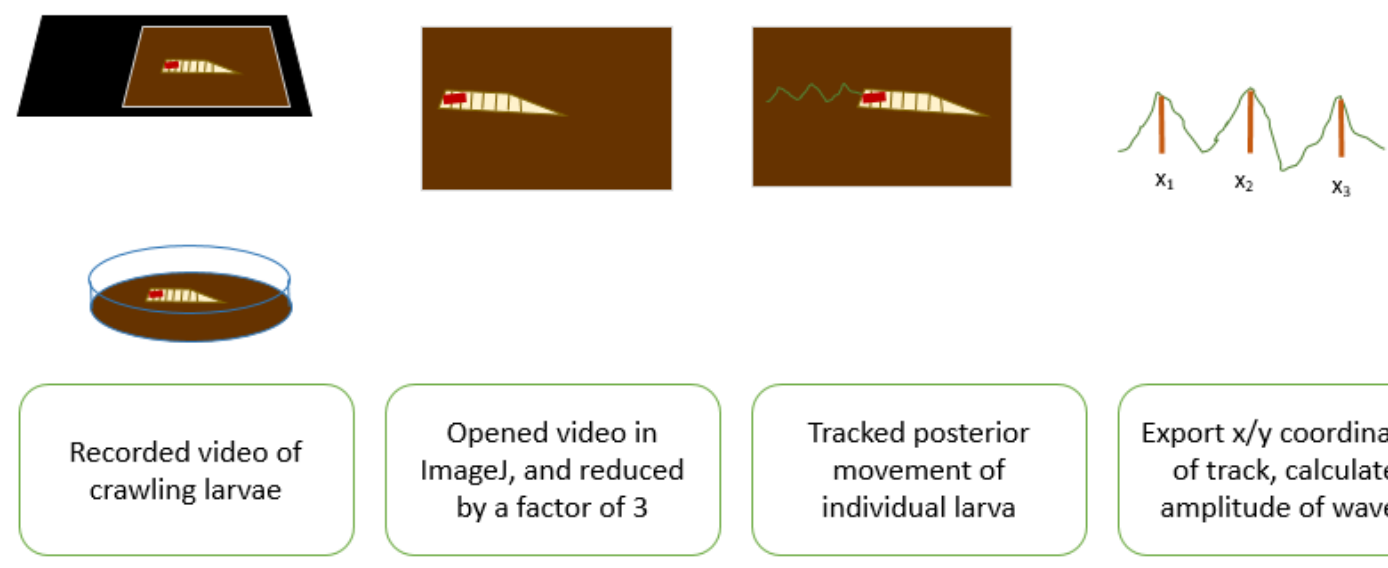
Tracked posterior movement of individual larva

Export $\mathrm{x} / \mathrm{y}$ coordinates of track, calculate amplitude of waves

Fig. 2.1 - Schematic of the collection and analysis process for assessing larval locomotion. Videos were recorded of larvae crawling in a standard molasses agar plate. Videos were then opened and processed in ImageJ, where the movement of the posterior end of the larva was tracked with the manual tracker plugin. The $x / y$ coordinates that were the output of manual tracker were then used to calculate the amplitude of the waves (amplitudes indicated with red line, and $x$ ). These values were averaged to produce a single value for each larva.

\section{Pharmacological Manipulations}

Serotonin levels were either increased or decreased in this assay through the use of pharmacological manipulations. To increase serotonin, larvae were fed 5hydroxytryptophan (5-HTP), which is a serotonin precursor. 5-HTP was fed to larvae mixed with yeast paste at a concentration of $2.5 \mathrm{mg} / \mathrm{ml}$. The food was prepared by dissolving 5-HTP in water with food coloring, and then mixing with an equal volume of yeast powder. Young foraging third instar larvae were selected and placed on the food for 24 hours. Larvae that were moving after 24 hours, with food 
coloring present in their digestive tracts were selected for use in the locomotion assay. After that time, larvae were immediately assayed in a fresh plate. Decreasing serotonin was accomplished by feeding larvae 4-chlorophenylalanine (pCPA). This drug was also administered as above at $2.5 \mathrm{mg} / \mathrm{ml}$ and fed over a 24 -hour period.

RNAi-based screen for serotonin receptors

To assess the involvement of specific serotonin receptors in the adaptation process, we obtained UAS-RNAi stocks to decrease expression of each of the known serotonin receptors. Each RNAi line was crossed with elav-gal4 to decrease expression in virtually all neurons, and repo-gal4 to decrease expression in glia. The identity of the individual receptors was coded, and was unknown to me until the data had been collected. The experimental crosses and the parental genotypes were analyzed for macro adaptation, and micro adaptation.

\section{Temperature Shift}

To address the contribution of temperature to the adaptation phenotype, we exposed larvae in this assay to temperature shifts at 15 minute intervals during the assay (Fig. 2.2). Larvae were glued at room temperature, as described above, and allowed to remain at room temperature for 15 minutes. Videos were subsequently recorded. Larvae were them moved to an incubator at $30^{\circ} \mathrm{C}$, and remained for 15 minutes. Videos were recorded immediately after larvae were removed from the 
incubator. Larvae were then returned to room temperature, where final videos were recorded after 15 minutes. The temperature shifts were 15 minutes long to ensure that the molasses agar plate that the larvae were crawling on reached the temperature of the environment, which we were able to measure with a thermometer.

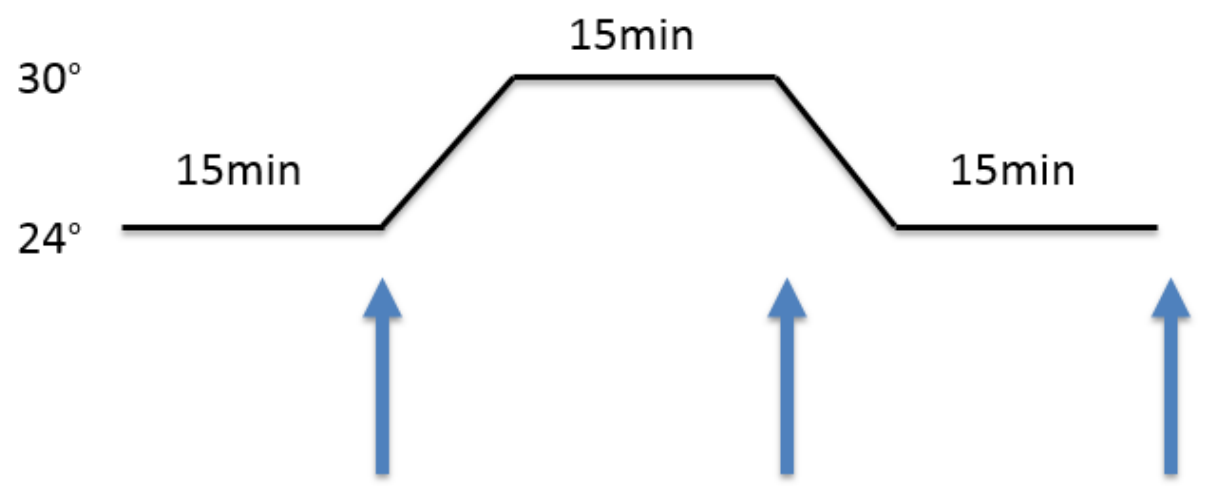

Fig. 2.2 - A diagram of the temperature shift scheme used to address the contribution of temperature to adaptation. Larvae were moved from 24 degrees Celsius to 30 degrees Celsius, in 15 minute shifts. Blue arrows indicate where data points were recorded, after larvae had been at that temperature for 15 minutes. 
Chapter 3 - The larval sensorimotor system exhibits plasticity in response to partial immobilization 
Abstract

Locomotion requires specialized neural circuits called central pattern generators (CPGs) that tightly control the movement of specific body parts. These circuits control crawling in a potentially changing environment, and in the presence of possible injuries. How these CPGs respond to localized disruptions is not well understood. To address this, we attached a splint to two adjacent abdominal segments on the right side of the larva, and observed their crawling behavior. The splint initially caused an exaggerated side-to-side movement of the posterior, characterized by a difference in compression timing between the left and right sides of segment A5. After four hours, the side-to-side movement was reduced and the left and right of $\mathrm{A} 5$ compress at the same time. Larvae are able to deadapt over a very short period of time after the splint was removed. These results indicate that changes in segment connections are capable of occurring in response to different stressors. In addition, we describe an assay that can be used to understand neuromodulators, genetics, and anatomical components of plasticity and change in CPGs and sensorimotor circuits.

\section{Introduction}

Neuroplasticity is broadly defined as the ability of the nervous system to change. The nature of these changes can be grouped into roughly two categories, synaptic and non-synaptic. Synaptic changes occur when the number, strength, or location of synapses are changed to modify the output of the circuit. In general, 
these changes result in synchronization of firing groups of neurons, or uncoupling pairs of neurons which had previously fired together. Non-synaptic changes in the context of neuroplasticity typically involve modifying the excitability of specific components of the circuit, to make them more or less likely to fire with a given stimulus, or to respond to a lesser degree.

Plasticity is a necessary feature of neural circuits that allows for them to respond appropriately and to carry out their function in different environments. As described in the introductory chapter, sensory neurons in circuits provide the sensorimotor circuit with relevant sensory information which can maintain circuit function in different conditions. One example worth describing is the action of chemosensory neurons in the brain, which influence the rate of respiration. When these chemosensory neurons detect increased levels of $\mathrm{CO}_{2}$, the circuit responds by increasing the rate of respiration to reduce the level of $\mathrm{CO}_{2}$ and to increase the level of oxygen.

In Drosophila, similar means of regulation are hypothesized to exist in conjunction with sensorimotor circuit. Experiments by Song (2003) and Hughes and Thomas (2007) demonstrate that altering sensory neuron activity does have an impact on crawling. Silencing specific groups of neurons often causes locomotion to slow, or results in abnormal segment compression (Hughes and Thomas, 2007). In the case of MD neurons, silencing all of them completely cause locomotion to stop, suggesting an incredible importance for that group of neurons (Song, 2003).

An interesting gap in the current body of research is how the sensory neurons in an individual segment contribute to locomotion. In all of the studies 
mentioned up to this point, groups of sensory neurons have been manipulated in all segments, using activation with channel rhodopsin or inhibition with shibire ${ }^{T S}$. One of the only studies to address how activity in one segment can influence other segments, is by Fox and Wu (2006). In this paper, Fox and Wu measured electrical activity in the nerve bundles going to different abdominal segments. They measured the same segments on left and right (bilateral), as well as adjacent segments on the same side (unilateral), and compared their firing patterns relative to one another. They found that when they cut a nerve bundle posterior to the neurons that they were recording, the activity in the other segments were disrupted. Anterior segments compressed at the inappropriate time, as well as in erratic patterns. These data suggest that feedback from a single abdominal segment does influence the activity of neurons in other segments (Fox and Wu, 2006). However, it should be noted that these experiments were conducted in semi-intact preparations, no in moving, crawling larvae. How crawling larvae respond to disruptions in individual segments is not well understood. In addition, these preparations are only viable for short periods of time. It would be virtually impossible to observe any potential changes that could occur in these larvae over prolonged periods of time.

To address the contribution of individual segments to crawling, we developed an assay in which we splinted two abdominal segments of third instar Drosophila larvae, and observed them as they crawled. We observed that, initially after the attachment of the plastic splint, larvae crawled with an exaggerated posterior movement. This movement could be attributed to asynchronous movement in segments immediately anterior to the plastic splint. This behavior was 
corrected four hours after the splint was attached, and the adaptation was reversible after the removal of the splint. This chapter describes the development of a novel assay, as well as a contribution to our knowledge of how segments influence each other's activity. In addition, we have found that the larval sensorimotor circuit displays the capacity for plasticity in the presence of the immobilization of specific abdominal segments.

\section{Results}

Immobilizing individual segments causes a unique crawling pattern, which is corrected after 4 hours

A simple model for understanding how Drosophila larvae propagate peristaltic waves is that each abdominal segment is controlled by only its own CPG. When a peristaltic wave is initiated in the posterior end, the motor neurons in that segment fire in A7, the posterior-most segment, causing A7 muscles to compress. As each individual segment compresses, mechanosensitive sensory neurons indicate compression of that segment, which simultaneously signal the silencing of the motor neurons in A7, and activate the CPG in segment A6. This simple mechanism controls when segments compress, how long they remain compressed, and when they begin to relax (Song 2007). Because the compression of an individual segment has been shown to influence CPG activity, we hypothesized that preventing the compression of a segment should influence the propagation of the peristaltic crawling wave (Fig. $3.1)$. 


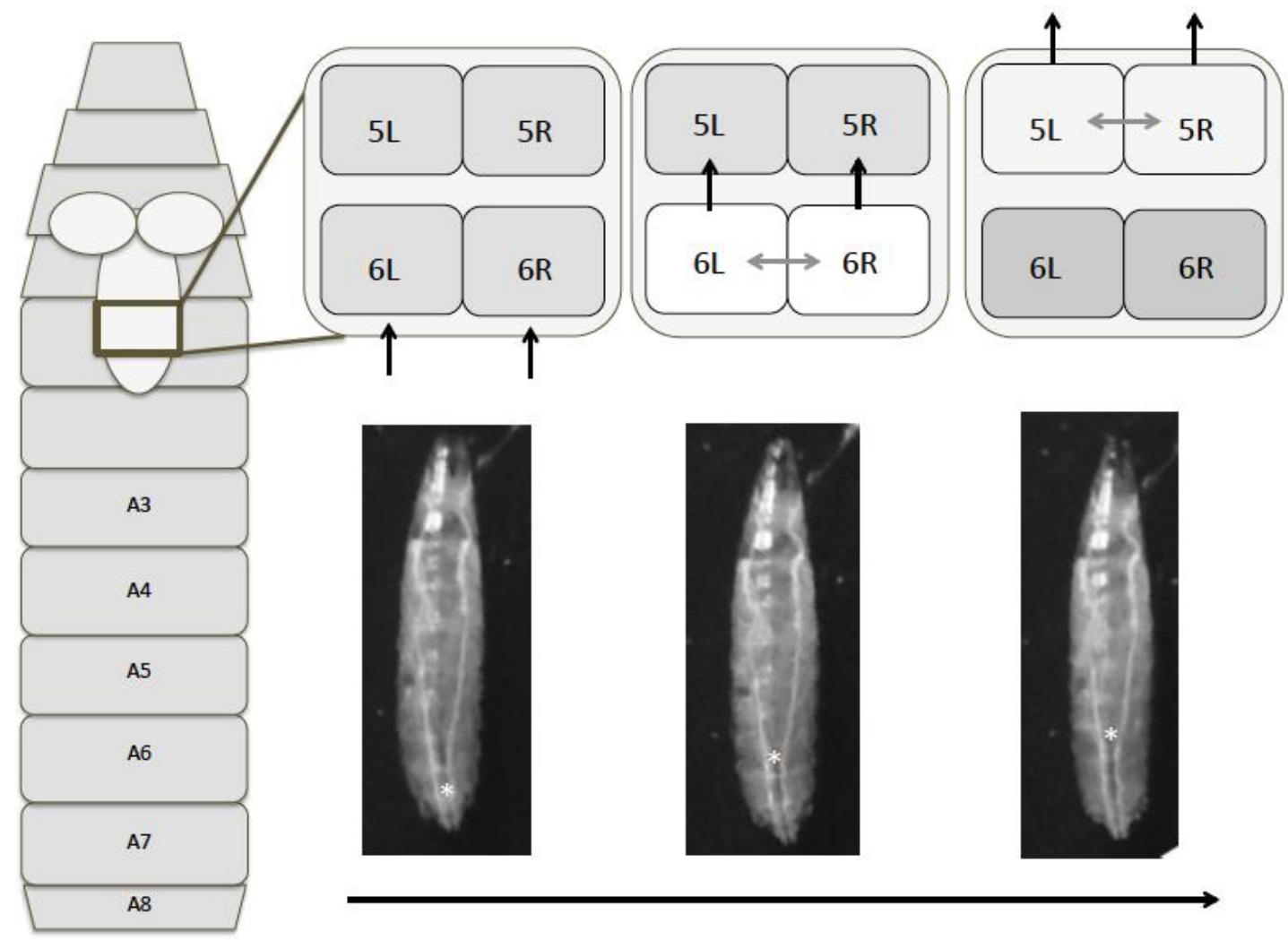

Fig. 3.1 - Larvae crawl using a series of interconnected CPGs, each controlling the left or right of a specific abdominal segment. These circuits are located in the VNC (shown inside the larval diagram). When segment $A 6$ receives input by an initiation signal (black arrows) (first panel), it signals activation of the CPG (light grey box). When this segment compresses, segment A6 sends a signal to A5 (middle panel), causing it to initiate compression (final panel). This is the general model for how segment CPGs propagate peristaltic waves in forward locomotion. In addition to connections causing feed-forward propagation, additional signals have been hypothesized to exist between left and right CPGs, detecting symmetry within a given segment (denoted with light grey arrows). 
To test this hypothesis, we devised a novel splinting assay. In this assay, we attached a plastic splint (see methods for specification) to the right side of a third instar larva, covering only the two most posterior segments (A6 and A7) (Fig. 3.2A). Immediately after splint attachment, larvae were placed an agar surface and allowed to crawl freely for 4 hours (240 minutes). Videos of larval crawling were recorded prior to splint attachment, as well as at several intermediate timepoints throughout the duration of the assay for the duration of the assay (Fig. 3.2B). In larvae with no splint attached, larvae crawl with their bodies in a straight line throughout the duration of the peristaltic wave. In contrast, in larvae immediately after the splint was attached, we observed a distinct side-to-side crawling pattern (Fig. 3.2B, 15min). We quantified this movement by measuring the movement of the posterior end of the larva (Fig. 3.2B, traces) and measuring the amplitude of the traces (white bar indicates measured amplitude) (Fig. 3.2B). Fifteen minutes after splint attachment, larvae had a three-fold higher height amplitude of posterior end swinging when compared to controls. However, intriguingly, this altered crawling behavior was absent from these same larvae just 4 hours later and the amplitude of side-to-side movement decreased to a value not statistically different to larvae without a splint attached (values quantified in Fig. 3.2C). These data indicate that when individual body segments are immobilized, it creates an altered crawling pattern that still enables the larvae to crawl. However, this pattern disappears after approximately 4 hours. These data led us to hypothesize that there is inherent plasticity. There were no observable differences in distance larvae crawled over the duration of the locomotion assay. 


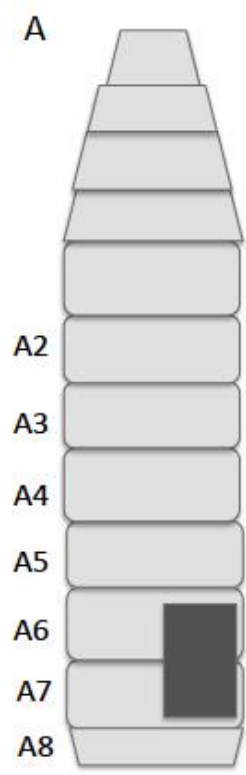

B

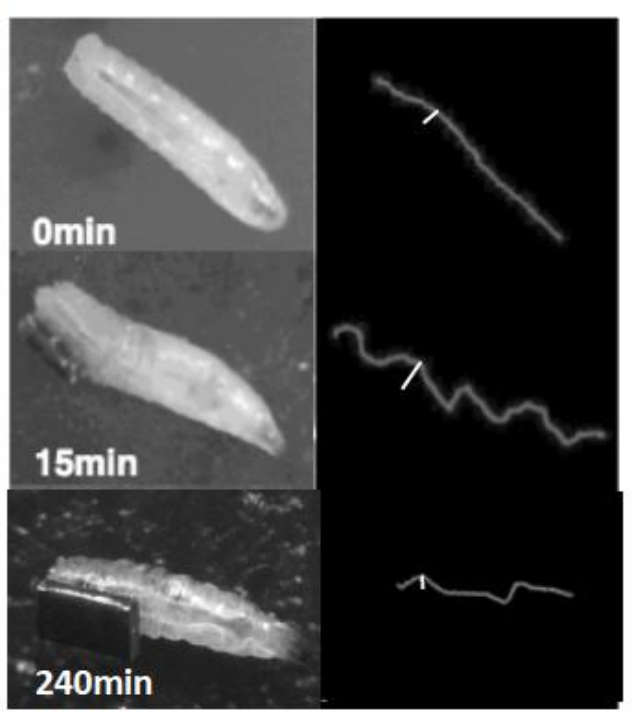

C

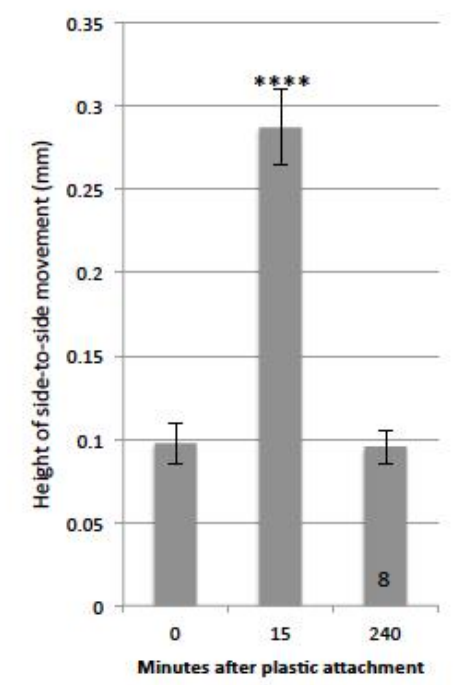

Fig. 3.2 - Larvae adapt in a sensorimotor plasticity assay. A) Diagram of a third instar larva showing the site of plastic attachment. The plastic splint (dark grey box) is attached to the right side of segments $A 7$ and A6. B) Images of larvae crawling with plastic attachment, with representative traces of posterior movement used for analysis. Omin (prior to splint attachment). The white bars show the amplitudes of the posterior movements, which were taken as a measurement for the asymmetric movement. C) Quantification of the amplitude of posterior movement during crawling. The posterior swinging amplitude is highest 15 minutes after plastic attachment (**** $=p>0.001$ ). After 4 hours (240min) the amplitude decreases to initial levels (ns different to Omin). 
$\underline{\text { Asymmetric segment compression results in errors in peristaltic wave propagation }}$

The current model of larval crawling describes that segments compress one at a time in an anterior-to-posterior fashion (Song, 2007; Heckscher, 2012). A potential source of the side-to-side movement we observe in our splinting assay could be that segments are compressing abnormally in response to the addition of the splint.

Using the assay described above, we carefully measured when the left and right sides of each abdominal segment were compressing in larvae that were splinted or unsplinted. In a video of crawling larvae, we considered the first frame a segment shortens to be the beginning of compression for that hemisegment. We considered a segment to be finished compressing in the first frame where the segment increases in length, or relaxes. To account for variation in crawling speed, the time points taken were normalized to the entire compression cycle (starting when segment A7 began to compress, and ending when segment A3 began to compress). Larvae with no splint attached consistently compressed and relaxed the left and right sides of each segment at the same time (Fig. 3.3B). However, in larvae with a splint attached to A6 and A7, we observed a significant difference between the initiation and cessation of segment compression in segment A5 (Fig. 3.3C). We observed a delay between when the left and side of A5 compressed and when the right side compressed. In fact, the right side of segment A5 compressed at the same time as the left side of segment A4. A phenomenon we never observed in unsplinted larvae. We observed this compression timing difference in A5 for approximately 2 hours into the assay (Fig. 3.3D). Interestingly, as larvae approached 4 hours in this 
assay and began to crawl normally, we no longer observed the compression timing difference in segment A5 (Fig. 3.3E). This suggests that the basis for the asymmetric crawling pattern is asymmetric segment compression in segment A5.

After attaching the splint, we observed timing delays in segments A4 and A3 in all splinted larvae (supplemental, fig. 3.1). However, the largest difference we observed over the course of the assay was in segment A5. For this reason, we hypothesize that the side-to-side movement induced by attaching the splint is corrected for by changing the timing of the compression of segment A5.

A

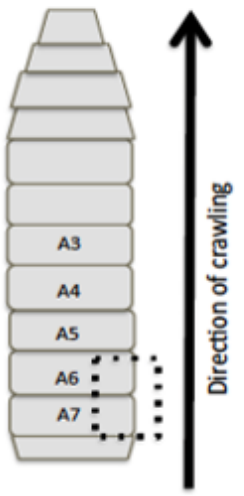

B

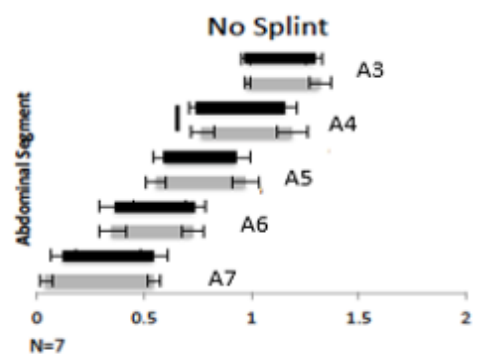

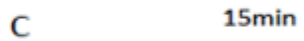

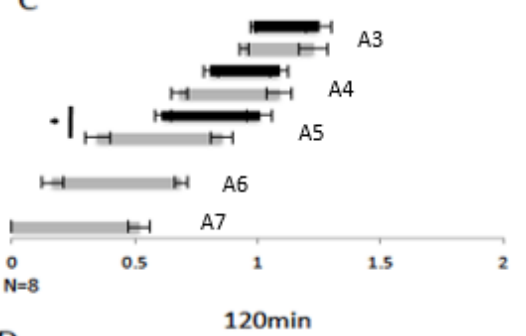

D

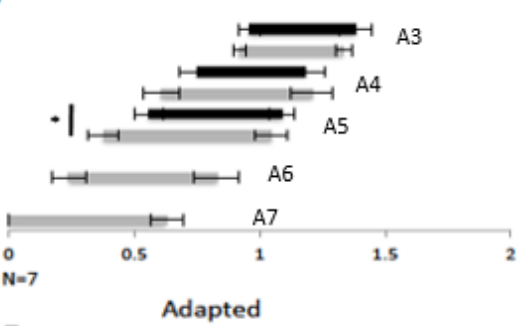

E

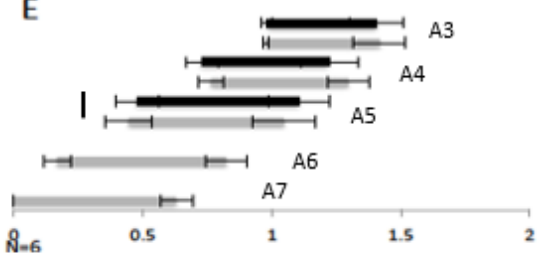


Fig. 3.3 - Splinting the right of A6 and A7 results in asynchrony between the left and right of anterior segments. A) Schematic of where splint was attached in this assay. B) Forward crawling larvae compress the left and the right of each segment at the same time. Segments compress in a posterior-to-anterior fashion. C) After attaching the splint to segments $A 6$ and $A 7$, we observe a delay between the left and the right of segment A5. D) The delay persists in larvae 2 hours after adaptation (120min). E) After 4 hours, the left and right of segment A5 compress at the same time. Error bars represent the difference in time compressed between the left and right of A5, black bars show non-significant differences. ${ }^{*}=p<0.01$.

Immobilized segments are compensated for with changes in surrounding body $\underline{\text { segments }}$

Attaching the splint in our assay caused a distinct crawling phenotype characterized by abnormal side-to-side movement in crawling larvae and asymmetric segment compression in A5. However, a question that remains unanswered is how the changes in segment compression are controlled. In the current model described by the literature, the compression of a specific segment results in compression of the next anterior segment (Fig. 3.1). However, in our assay, both sides of segment A5 are able to compress, although asymmetrically, without normal compression of segment A6. Therefore, we hypothesize that there are alternative ways to initiate compression in the right of A5 that are independent of segment A6 and its CPG. Based on the timing of segment compression observed in 
splinted larvae, it is possible that the right side of segment A5 is compressing in response to the left side of segment A5 compressing. The right side of A5 and the left side of A4 are compressing at the same time, which is consistent with a common signal causing their compression. If the left side of A5 is providing the signal causing the right side of $\mathrm{A} 5$ to compress, then hindering the left side of A5 compression should prevent the compression of the right side of A5.

To test this hypothesis, we repeated the assay described above, and then attached a second splint to immobilize segments A4 and A5 on the left side of the larvae (Fig. 3.4A). As a control, we repeated the assay with the second splint attached to the left side of segments A4 and A3, leaving segment A5 left free to compress (Fig. 3.4B). When the experimental group of larvae were observed, the majority of them ( $\sim 80 \%)$ were unable to crawl (Fig. 3.4C, "A5 Splinted"). These larvae were all observed trying to initiate a peristaltic crawling wave (characterized by movement of A7), but they were unable to compress in the segments anterior to the plastic splint. The small percentage of larvae that were able to could reflect variation in how individual larvae crawl, which has not been quantified by any additional means. Larvae in which segment A5 was allowed to compress, but were immobilized in A4 and A3, were able to crawl normally (Fig. 3.4C, "A5 Unsplinted"). Taken together, it does appear that the majority of larvae rely on the compression of the left side of A5 for the compression of the right side of segment A5.

In larvae with one splint attached, we observed a gradual change in the timing of compression in individual segments. In larvae that had adapted and reduced their posterior side-to-side movement, we observed a change in the timing 
of segment compression. One explanation for the shift in compression timing that we observe, is that the signal required to activate segment A5 has shifted. Our results so far would support the hypothesis that the left side of segment A6 is providing the compression signal required for both the left and the right side of A5 to compress. If this is the case, larvae with segment A5 immobilized after they have adapted to the initial splint should be able to execute a peristaltic wave. Indeed, this is what we observe in larvae where segment A5 is attached (Fig. 3.4C, "A5 splinted (adapted)"). These results indicate that, although the left side of A5 is required initially for the peristaltic wave to propagate, it is not required for compression in larvae that have adjusted their segment compression pattern.

A

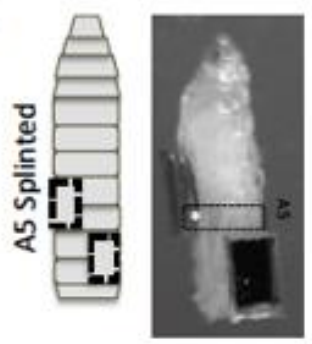

B

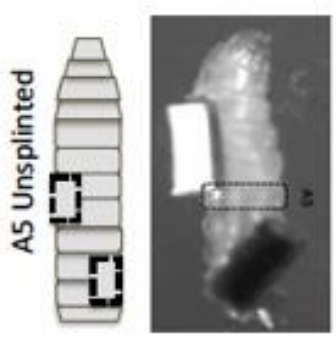

D

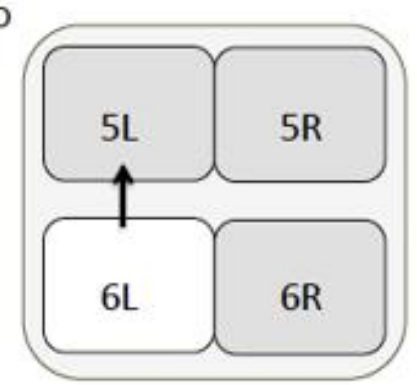

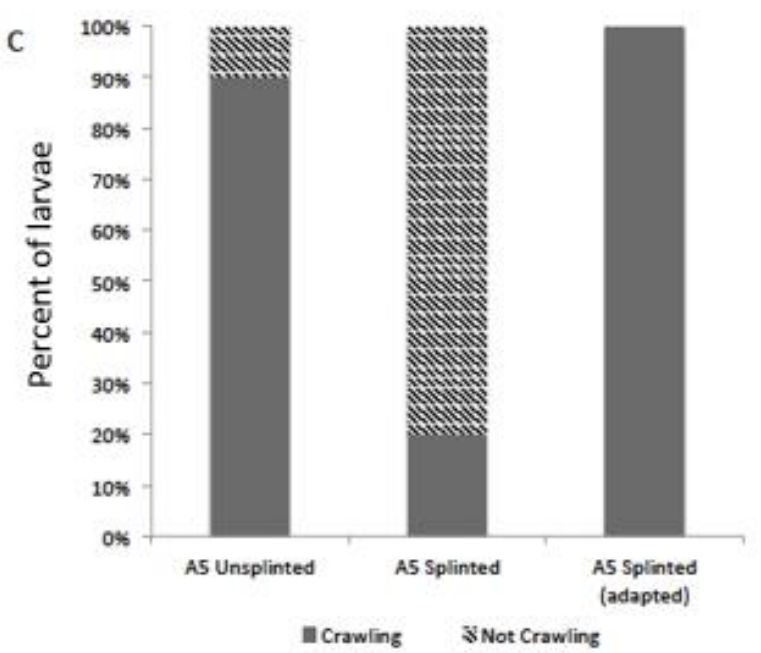

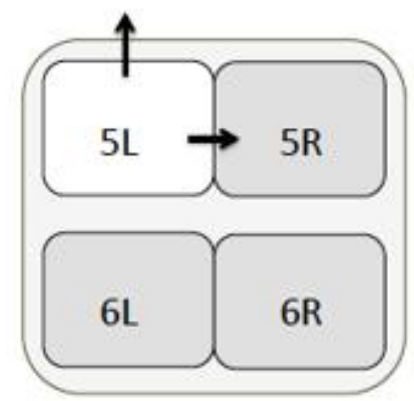


Fig. 3.4 - Compression of the left side of A5 is required for peristaltic wave propagation in the presence of the original splint. Larvae in this assay were splinted with two splints. The first splint was attached in the same location as previously described (the right side of A6 and A7), but then a second splint was attached on the left side of anterior segments in one of two conformations. A) Several larvae were attached with the second splint covering the right side of $A 4$ and A5. B) The remaining larvae were splinted with the second splint attached to segments A3 and A4, leaving segment A5 free to compress. White asterisks indicate whether the left side of segment A5 is covered or not. C) Larvae with A5 free to compress were able to carry out normal peristaltic waves. However, larvae with the left side of segment A5 splinted were unable to carry out peristaltic waves. However, if the second splint was attached to the left of A5 after larvae had already undergone adaptation, larvae were able to crawl. Each category contains 8-10 larvae observed. D) These data support the model that initially in the assay, the left side of segment $A 5$ needs to compress in order to initiate the compression of the right side of segment $A 5$.

\section{Changes in segment connectivity are reversible}

Data presented thus far describe changes to forward crawling patterns of Drosophila larvae. A possible hypothesis for the changing behavior is that the neural circuits governing compression of each hemisegment are changing in response to the immobilization of specific segments. Although the exact location and nature of these changes is unclear, we wanted to understand whether the changes that we observed were permanent or reversible. If the pattern of compression that we 
observed is reversible, we would expect to see a return to the pattern of segment compression observed in larvae prior to the addition of the splint.

To test this, we allowed larvae to adapt normally in the assay, and subsequently removed the splint. We observed larvae immediately after the splint was removed, and every 5 minutes following. Although the asymmetric side-to-side movement did not increase significantly above what was observed in adapted larvae (Fig. 3.5A), we did observe differences in how segments were moving. Initially, the left side of segments A6 and A7, which had previously been covered by the splint, were much slower to contract than their contralateral sides (Fig. 3.5B). However, the left and right sides of segment A5 continued to contract in sync as they had before the splint had been removed. After approximately 15 minutes, each segment once again demonstrated coordination between the left and right sides (Fig. 3.5B). Because both halves of A5 are in sync throughout the deadaptation process, it appears that if the left side of A6 is providing the source of compression for both the left and right of A5, it continues to do so even after the plastic is removed (Fig. 3.5C). It remains unclear whether the potential circuit changes seen early in the assay are persisting after the larvae have deadapted, but it is clear that the segment compression dynamics have returned to normal after the removal of the plastic. 

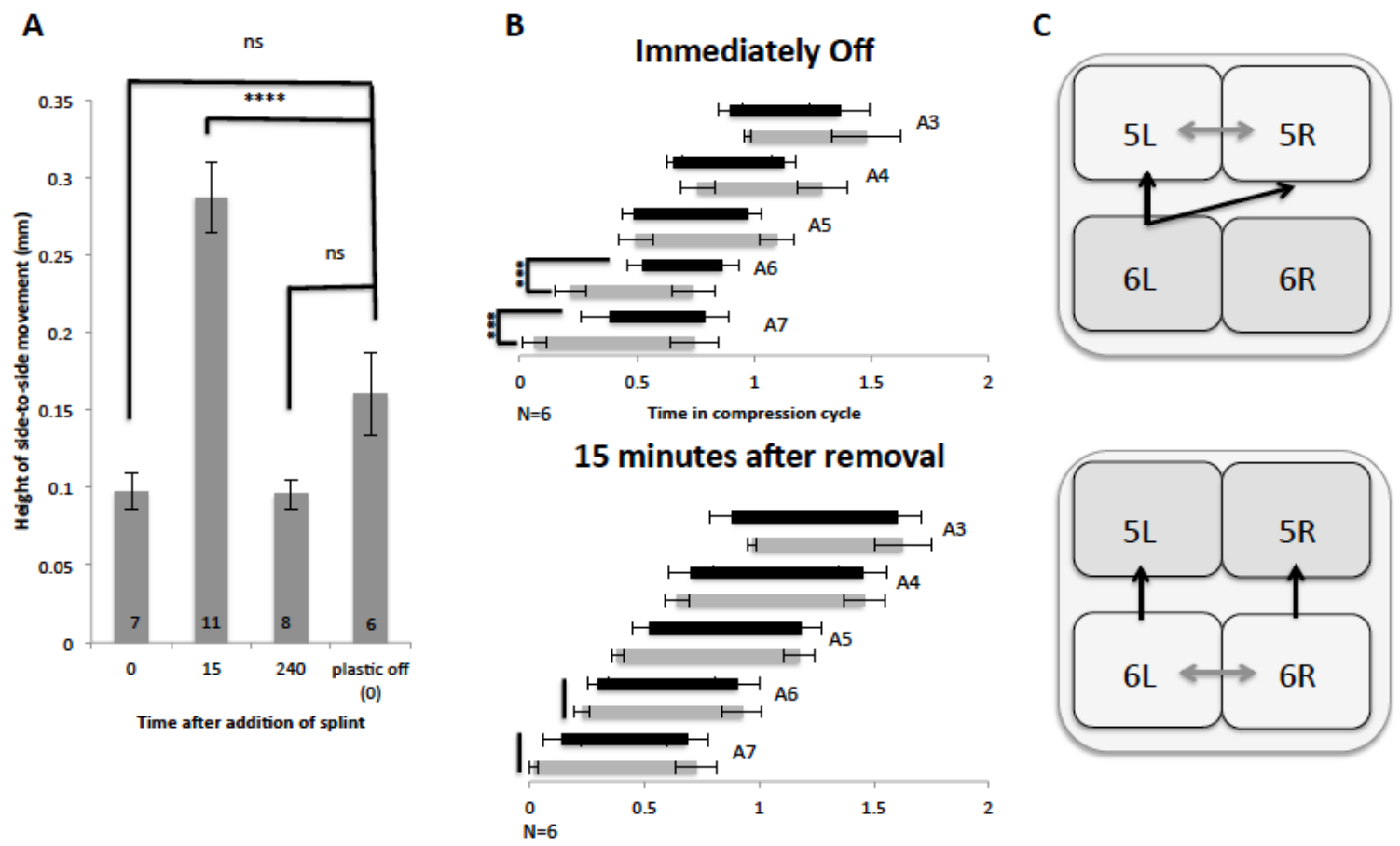

Fig. 3.5 - After plastic removal, the segment the pattern of segment compression reverts to the pattern observed in wild type larvae. A) General side-to-side movement in the posterior end was not statistically different immediately after the plastic is removed. $B$ ) When the segment compression timing was measured, initially ( $0^{\prime}$ off) the left of $A 6$ and $A 7$ were delayed relative to the right sides of the same segments. 15 minutes after the plastic is removed, the delay disappears and has returned to compress at approximately the same time. C) A model of what is occurring after the splint is removed. Initially, the compression of segment $6 \mathrm{~L}$ is capable of initiating the compression of both $5 \mathrm{~L}$ and $5 R$. Segment $6 \mathrm{~L}$ is likely still being stimulated to contract by segment $7 L$ (not shown). However, by 15 minutes after the plastic has been 
removed, segment 6 R generates the signal responsible for initiating the compression of 5R. $N=6\left({ }^{* * *}=p<0.01\right)$, black bars represent non-significant differences.

Based on the timing of segment compression observed when one splint was attached, we hypothesized that the signal required to initiate compression of segment A5 was coming from the compression of the left side of A6. To test this, we attached a second splint to the larvae after they had adapted, preventing the compression of segments A6 and A7 on both sides (Fig. 3.6A). After the splint was attached in this conformation, larvae were still able to crawl with the segment compression dynamics observed with one splint (Fig. 3.6B). This indicates that larvae do not depend on the left of A6 in order to generate compression of A5. This could mean one of two things. One possibility is that the left of A6 is now taking over as the compression signal required in adapted larvae. A second possibility is that the left of A6 is capable of generating the compression signal for A5, but the original connection between the left and right of A5 is still intact and functional in the absence of activity in A6. Our data more strongly support the conclusion for the former hypothesis, but more experimentation is required to examine the second possibility more closely. 
A

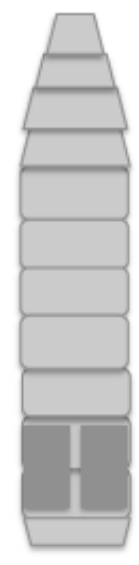

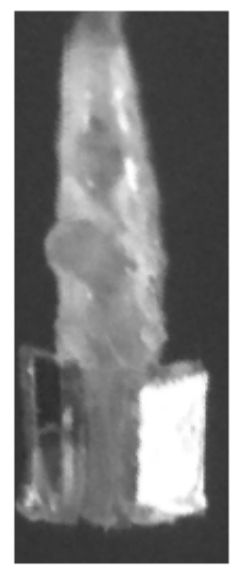

B Attached after adaptation
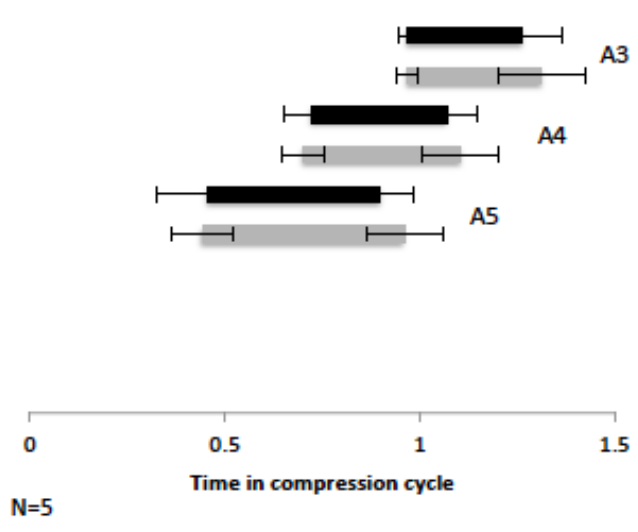

Fig. 3.6 - Compression of segment A6 is not required for compression in larvae that have already adapted. A) In this assay, a second piece of plastic was attached to the left side of A6 and A7. B) Larvae are able to crawl and compress their segments at the same time.

Canton S larvae do not examine the same phenotype observed in Oregon R larvae

Several wild-type strains of Drosophila are commonly used in behavioral experiments. In the experiments described here, OR larvae were used. However, Canton S (CS) is an additional strain of Drosophila which are often used as a genetic background for behavioral and genetic studies. Although OR and CS are often used interchangeably within the field, it is important to consider the possibility that differences in genetic background could play a role in our adaptation phenotype. Researchers using mice as an experimental system have long understood this: phenotypes of genetic knockouts as well as pharmacological treatment vary widely based on which background strain of mouse is being used (Wolfer, 2002). To address this, we performed the assay in CS larvae. CS larvae exhibited a different adaptation phenotype than that observed in OR larvae (Fig. 3.7). However, when 
compared to other genotypes (SN-GFP), the pattern of segment compression in OR looked more like the OR behavior than the CS behavior. We therefore concluded that the adaptation phenotype observed in OR are the more commonly observed adaptation pattern.
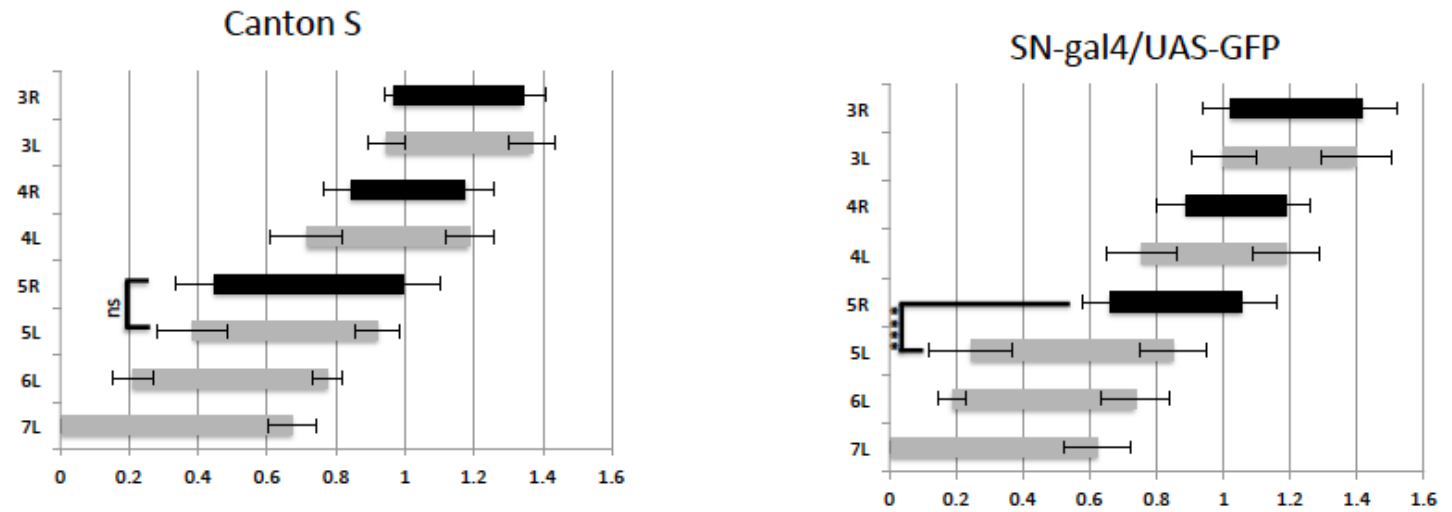

Oregon R

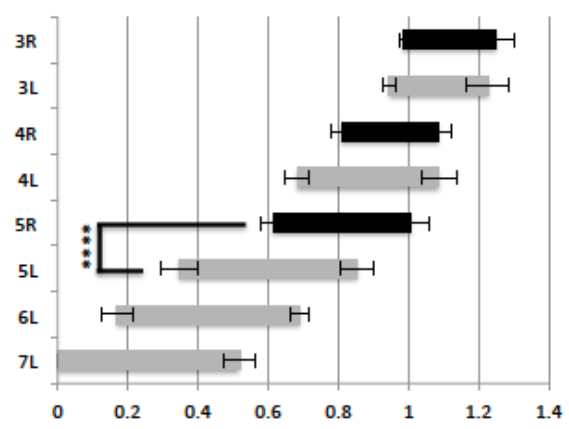

Figure 3.7 - Canton S larvae respond differently to the presence of a splint than either Oregon R or SN-gal4/UAS-GFP.

Larval crawling is controlled by a series of interconnected CPGs that rely on sensory feedback in order to propagate (Song, 2007; Kohsaka, 2012). Compressing segments at the right time and in the right order is necessary to properly execute specific crawling patterns, including forward crawling, reverse crawling, and turning Several neurons and neurotransmitters have been identified which are necessary for 
ensuring that specific motor behaviors occur in the appropriate context (Caldwell, 2003; Song, 2007; Heckscher, 2012; Okusawa, 2014). With so much machinery required to control behavioral output, this system is likely sensitive to asymmetric inputs to the circuit. To test this, we developed splinted larvae on one side of their abdominal segments and asked if crawling behavior was altered and how. Larvae with a splint attached developed a unique side-to-side movement in their posteriors. After approximately 4 hours, this side-to-side movement has been reduced.

The source of the posterior movement can been attributed to differences in the compression of the left and right sides of A5. Early in the assay, the right side of segment A5 is significantly delayed, so much so that it is now compressing at the same time as the left side of segment A4. Because the right side of A5 normally receives the signal to initiate compression from the right side of A6, this leaves two possibilities for how compression of the right of A5 is occurring. The first possibility is that the right side of segment $\mathrm{A} 6$ is able to compress enough under the splint to activate the right of A5. This possibility seems unlikely, because in the sets of experiments in which a second splint is attached covering the left of A5, the peristaltic wave is no longer able to propagate. A second and more plausible possibility is that when the left side of A5 compresses, it sends an initiation signal to the right side of A5. Such a connection between segments has been proposed in the modeling study by Gjorgjieva (2013), although anatomical presence has not been confirmed. In order to begin to understand the changes that we are observing, a lot can be learned by simply examining the reversibility of the behavior. In this assay, adaptation after the splint is attached occurs in approximately 4 hours. In 4 hours, a 
number of changes could be occurring in order to cause the changes in crawling pattern. For example, neurite growth in the CNS could be occurring, and physically causing a connection to form between the left side of A6 and the right side of A5. Alternatively, new synapses could be developing by either inserting or removing receptors in the membrane. The data here do not suggest one change or another, but both options may be explored in the future. What is fascinating, is that deadaptation, or reversing the changes, takes only about 15 minutes. Because this change is so rapid relative to the initial adaptation, we hypothesize that after the splint is removed there are rapid silencing of the synapses or neurite branches that had developed.

Although a neuromodulator is likely involved in this process, it isn't likely that there changes observed here are the result of simply rapid neuronal signaling. Serotonin has been shown to acutely suppress rearing and to allow for proper turning behavior (Okusawa, 2014). Tyramine has also been shown to rapidly cause changes in crawling speed in response to food deprivation (Koon, 2011). In both of these cases, the neurons generating these peptides are signaling to a downstream neuron, and either increasing or decreasing its activity and downstream neurons. Instead, we propose that the changes occurring in this assay are more like the changes observed in Knudsen's barn owl experiments (1989), where neuron circuit changes occurred gradually, reverted rapidly, and when tested later were found to persist (Knudsen, 1989). In this situation, we hypothesize that the change that is more likely occurring is the result of either altering the gain or sensitivity on a part of this circuit, or causing small and reversible changes to neuron structure. 
The identification of specific neurons and signaling components will be crucial to understanding the nature of this adaptation process. In subsequent chapters, I will discuss evidence that we have gathered implication a potential role for temperature and serotonin in adaptation. However, understanding the specific neural components, and how they are changing to bring about adaptation is crucial. 
Chapter 4-Serotonin regulates adaptation in the larval sensorimotor circuit 
Abstract

Locomotion is a complex behavior, which is mediated by a tightly regulated group of neural circuits. Regulation at the level of individual neurons, as well as between groups of neurons, are crucial for ensuring efficient and productive locomotion. In Drosophila larvae, locomotion is carried out through the coordinated, sequential compression of a large number of abdominal segments which generate a peristaltic wave. Recently, we described an assay in Drosophila larvae which allowed us to investigate the role of individual abdominal segments to the compression of surrounding segments, and discovered that the circuit possessed the capacity for adaptation in response to changes in an individual segment. In this dissertation chapter, we wanted to examine the role of serotonin in the adaptation process. Larvae with elevated serotonin adapted much more rapidly compared to wild-type, and larvae with decreased serotonin levels failed to adapt by the same time as wildtype. Overexpression of the serotonin transporter SERT adapted the most rapidly of any of the genotypes tested. A directed RNAi screen reveled that serotonin is acting through the 5-HT7 receptor expressed on glia to facilitate adaptation. These data contribute to our understanding of how the larval segments communicate, and also how larvae are able to cause adaptation in the sensorimotor circuit. 


\section{Introduction}

Serotonin (5-hydroxytryptamine, 5-HT) is a neurotransmitter involved in many physiological processes. It is synthesized from the amino acid tryptophan, in a two-step synthetic process. Tryptophan is initially converted into 5hydroxytryptophan (5-HTP) by the enzyme tryptophan hydroxylase (encoded by the gene trh), and then finally synthesized into serotonin by the enzyme dopa decarboxylase (DDC). Trh is specifically expressed in serotonergic neurons, and can be used as a marker of serotonergic function, but DDC is also a crucial step in the synthesis of dopamine, and is therefore also found in dopaminergic neurons.

A portion of the serotonin released into the synapse is taken up by the serotonin transporter SERT (dSERT in Drosophila). This is a serotonin-specific transporter, and it is incredibly important for replenishing the pool of serotonin available at the synapse, and limiting its activity on post-synaptic neurons (Borue, 2010). Because SERT removes serotonin from the synapse, it is the pharmacological target of disorders in which serotonin is misregulated. Selective serotonin reuptake inhibitors (SSRIs) are used to treat depression, as well as disorders of addiction. Blocking SERT activity causes increased persistence of serotonin in the synapse, allowing more of it to reach its postsynaptic target. The most familiar SSRIs used are citalopram, fluoxetine, and sertraline, which are used to treat depression, eating disorders, generalized anxiety, and obsessive compulsive disorder (reviewed in Beasley , 1992). Although these drugs are considered to be very beneficial, and are very widely described, there are side-effects which provide insight into the additional role that SERT may play in physiology. Some of the widely-reported side 
effects observed are suicidal tendencies, digestive troubles, and sexual dysfunction. Serotonin is also very important for the regulation of these functions, described below.

On the postsynaptic membrane, serotonin can bind to one of 14 serotonin receptors in humans (4 possible receptors in Drosophila). Serotonin receptors (5HTRs) are almost all G-Protein coupled receptors (GPCRs), with the exception of 5HT3, which is a cation channel. Interestingly, all of the receptors that have been identified in Drosophila are GPCRs, no homolog of the 5-HT3 receptor has been identified to date. Serotonin has been shown to elicit a variety of downstream signaling cascades through its individual receptors.

Serotonin is of great medical interest largely because of its role in mood and depression. However, serotonin signaling is also a common pharmacological target for disorders involving gut peristalsis. Approximately $90 \%$ of the body's serotonin is produced in the gut by the enteric nervous system, intestinal enterochromaffin cells, and gut bacteria (Gershon, 1991; 1997; Yano, 2015). Disorders such as irritable bowel syndrome (IBS), constipation, diarrhea, and severe nausea are often treated with drugs targeting components of the serotonergic system. Several 5-HT3 receptor antagonists, including ondansetron and granisteron, are approved to treat chemotherapy-associated nausea. A different $5-\mathrm{HT}_{3}$ antagonist, alosteron and tegasteron, a partial $5-\mathrm{HT}_{4}$ agonist are both approved for the treatment of irritable bowel syndrome (IBS). Serotonin receptors are located on enteric sensory neurons, smooth muscle cells, and enterocytes in the gut. The number of receptors and cell types involved downstream of serotonin indicate that this process isn't as 
straightforward as an "on-off" switch, but involves delicate tuning of several network components.

Although serotonin has not been clinically applied to modify locomotor behavior, a large body of research supports the role of serotonin in controlling locomotion speed and gait in vertebrate and invertebrate systems. In zebrafish, serotonin is crucial for the development of neural networks that govern swimming. Serotonin is required very early in development to modulate swimming behavior, and is thought to induce episodes of spontaneous activity during the development of the sensorimotor circuit (Brustein, 2003). In the mammalian spinal cord, serotonin plays a role in controlling gait and limb coordination. When serotonin is applied to the exposed mammalian spinal cords, the excitability of motor neurons is increased via $5-\mathrm{HT}_{1 \mathrm{a}}$ receptors and $5-\mathrm{HT}_{2}$ receptors.

In Drosophila, several researchers have tried to examine the role of serotonin in locomotion of larvae. Anatomically, serotonergic neurons are found in pairs in each segment of the VNC, as well as in the brain. Serotonin can influence the rate of crawling (Dasari and Cooper, 2009), as well as the tendency to turn (Okusawa, 2014). Serotonin also plays a role in the integration of sensory information to control movement throughout their environment. Larvae require a functioning brain to navigate throughout their environment, and several of these processes require serotonin signaling. Serotonin signaling to receptors in the mushroom bodies in the brain are thought to also influence the direction of locomotion (Moncalvo and Campos, 2009; Silva, 2015). In addition to the mushroom bodies, neurons in the subesophageal zone contribute to the integration of sensory 
information (Tastekin, 2015). Although there are serotonergic neurons located here, there is currently no data to suggest that these neurons contribute to crawling.

While serotonin has been shown to influence locomotion, a mechanism for the action of serotonin has not been proposed or tested in the literature. If we draw from literature in vertebrates, one possible means of influencing the rate of locomotion is through efficient coordination of body segments to maximize the distance moved per peristaltic wave.

Based on what we know about vertebrate models, serotonin could very well be involved in controlling the rate frequency of locomotion as well as the coordination between the left and the right. The assay we described in an earlier chapter provides an excellent opportunity to ask this question, because it directly challenges coordination between neighboring abdominal segments. Our results indicate that serotonin increases the rate of adaptation observed in Drosophila larvae that have been asymmetrically splinted. The increased rate of adaptation is especially pronounced when serotonin is increased specifically at the synapse, which were accomplished with the overexpression of the serotonin transporter in serotonergic neurons. We also used directed expression of RNAi to the known serotonin receptors, and identified the 5-HT7 receptor on glial cells as the postsynaptic candidate for serotonergic mediation of the adaptation process. Taken together, these data point to serotonin as the primary regulator of plasticity in the larval sensorimotor circuit, by increasing the speed at which left and right hemisegments were able to re-coordinate. 


\section{Results}

Increased serotonin causes rapid adaptation

Because of the role of serotonin in permitting and facilitating adaptation in other neural circuits, we wanted to examine whether or not serotonin plays a role in the sensorimotor plasticity. To manipulate serotonin, we fed larvae 5hydroxytryptophan (5-HTP), which is the synthetic precursor to serotonin. Oregon $\mathrm{R}$ (OR) larvae were fed $2.5 \mathrm{mg} / \mathrm{ml}$ of 5 -HTP dissolved in food for 24 hours prior to experimentation. Larvae that had ingested food were selected for the assay, which we conducted as previously described. Larvae were fed over a 24-hour period to assure that they were ingesting the drug, but also to minimize any developmental defects that could result from prolonged manipulation of serotonin levels.

OR larvae that had been fed 5-HTP successfully adapted two hours after being splinted with the plastic (Fig. 4.1, blue bars). Adaptation was determined to have occurred when the amplitude of asymmetric crawling was statistically different than the level of crawling at 15 minutes, but not statistically different to the level of asymmetric movement observed in non-splinted larvae. This is two hours faster than Oregon $\mathrm{R}$ larvae which had not had their serotonin levels manipulated (Fig 4.1, gray bars). 


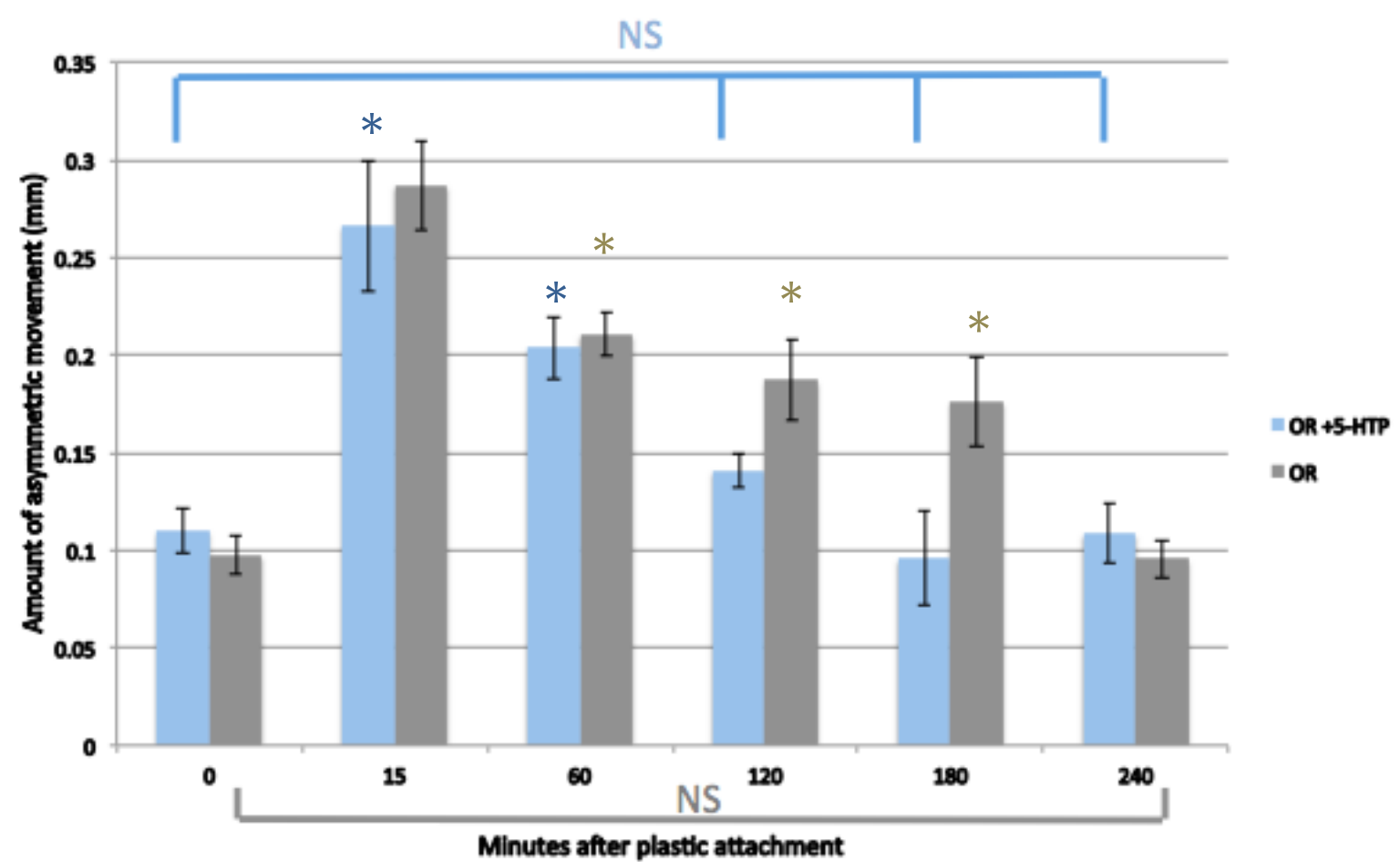

OR larvae treated with 5-HTP (blue) reduced their asymmetric crawling pattern by 120 minutes after plastic attachment. OR who were not treated with 5-HTP do not adapt until 240 minutes after adaptation (gray). $N=8$. Blue asterisks indicate a significant difference between the indicated time point, and the initial time point for the 5-HTP fed larvae. Gray asterisks denote the same statistical information for OR control. $N=8$ for OR larvae.

Inhibiting serotonin synthesis prevents adaptation from occurring

Our results above indicate that serotonin is sufficient to increase the rate of adaptation after the attachment of the splint. To test the necessity of serotonin for adaptation, we fed larvae p-chlorophenylalanine (pCPA), an inhibitor of serotonin synthesis. Larvae were fed for 24 hours, in the same time course as was conducted with the 5-HTP. After 24 hours, larvae that were still moving and had visibly 
ingested the food containing the drug were selected for the locomotion assay. It should be noted that, in general, fewer larvae were moving in the pCPA-fed plates as were in either the 5-HTP plates or wild-type. This is consistent with previously published observations that decreasing serotonin levels results in decreased motor neuron activity (Dasari and Cooper, 2005). These larvae never observed adapting in the locomotion assay. pCPA-treated larvae developed the same asymmetric movement that we observed in untreated larvae at 15 minutes. Four hours after attaching the splint, these larvae retained the same degree of asymmetric crawling that they displayed fifteen minutes after the addition of the plastic (Fig. 4.2, blue bars). Larvae were never observed to adapt at subsequent time points, however as time went on larvae eventually pupated or totally stopped moving, which made analysis of these larvae impossible.

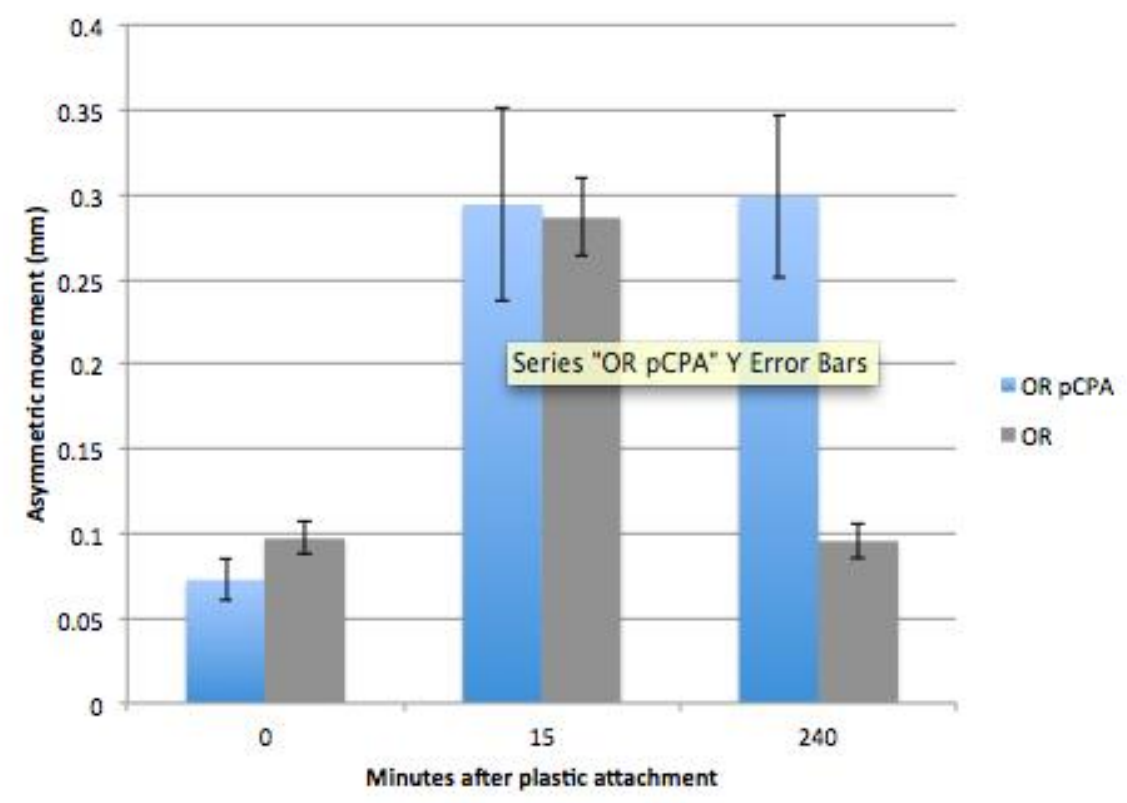

Fig. 4.2 - Decreasing serotonin synthesis prevents adaptation by 240 minutes. OR larvae treated with $p C P A$ (blue) failed to adapt by 240 minutes, as was observed in 
untreated OR larvae (gray). $N=6$. Oregon $R$ wild-type controls are shown in grey as controls. $N=8$ for OR larvae.

Overexpression of the serotonin transporter, dSERT, resulted in very rapid adaptation

As mentioned previously, a prominent means by which serotonin is regulated is through the activity of the serotonin transporter, SERT. SERT controls the persistence of serotonin at the synapse, and is very important for replenishing the serotonin pool at the synapse of the serotonergic neuron. Overexpressing dSERT in serotonergic neurons has been shown to selectively increase serotonin at the presynaptic junction of serotonergic neurons. As a result, overexpression should reveal the contribution of a large pool of serotonin to adaptation in our plasticity assay. To test this, we overexpressed dSERT in serotonergic neurons (Trh-gal4/UAS$d S E R T$ ), and performed the locomotion assay on them during the late foraging $3^{\text {rd }}$ instar stage. Unexpectedly, these larvae adapted much more rapidly than any other genotype that we have observed. The asymmetric crawling pattern was observed to be highest 5 minutes after the addition of the splint, and had completely diminished by 15 minutes (Fig. 4.3). 


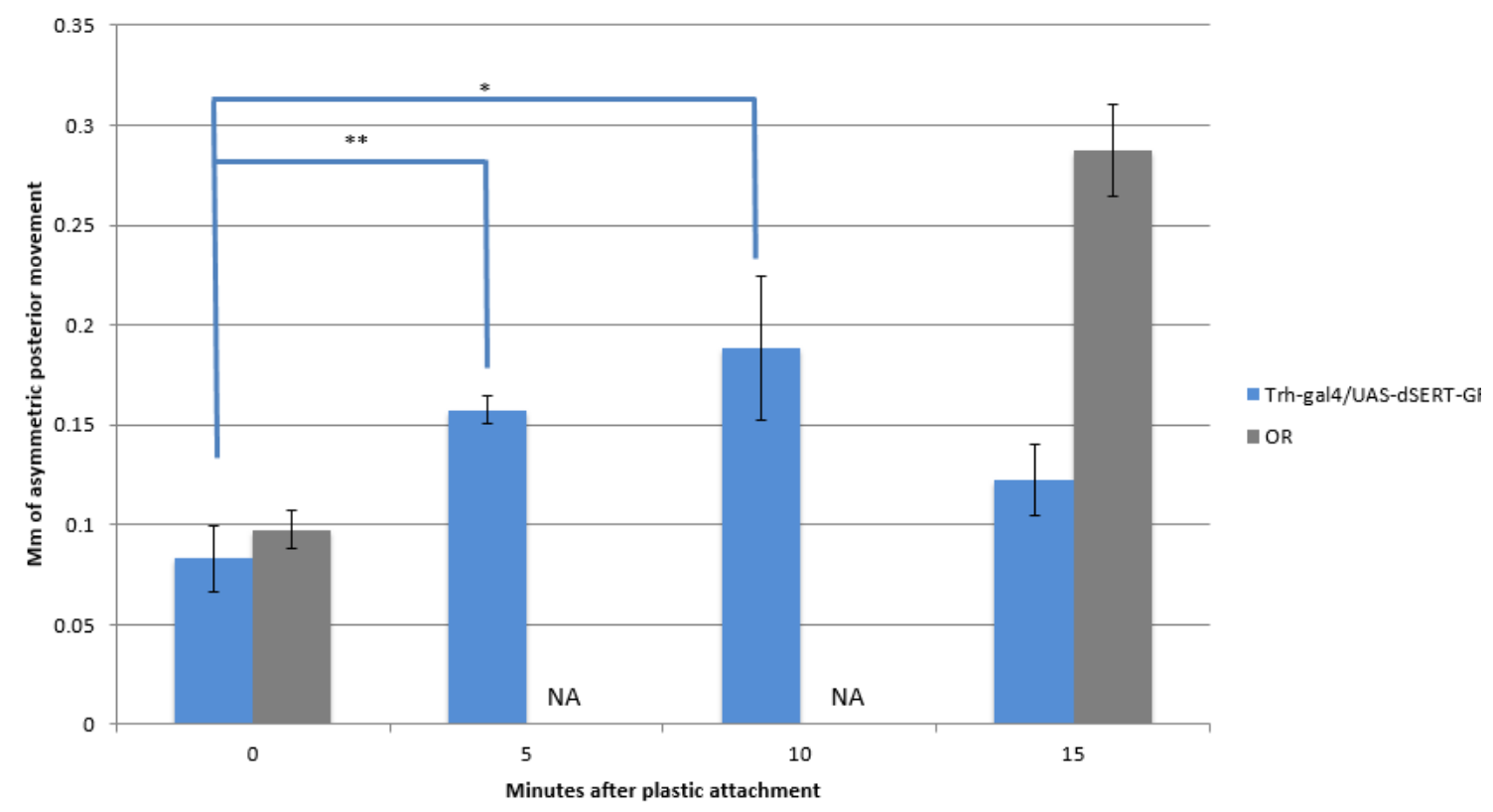

Fig. 4.3 - Overexpression of the serotonergic transporter in serotonergic neurons results in very rapid adaptation. Asymmetric crawling peaks in larvae by 5 minutes after the splint is attached, and they have successfully adapted by 15 minutes after the plastic was attached. Due to the nociceptive rolling behavior in wild-type larvae, we were unable to collect data for OR larvae at the 5 and 10 minute time points (indicated NA). The largest difference was observed at 5 minutes after the plastic attachment ( $\left.{ }^{* *} p<0.01 ;{ }^{*} p<0.05\right) . N=6$ larvae for Trh-gal4/UAS-dSERT.

Expression of the 5-HT7 receptor expressed in glial cells is required for successful adaptation.

Data above support the hypothesis that sensorimotor adaptation is controlled by serotonin. We have shown that increasing serotonin causes rapid 
adaptation, and that reducing serotonin prevents adaptation from occurring. If serotonin is controlling this behavior, we hypothesized that we should be able to identify the receptor subtypes acting downstream of serotonin to facilitate adaptation. We are also interested in whether serotonin was acting in neurons or glia in order to cause adaptation. To examine the role of individual serotonin receptors and where they were acting, we conducted a directed RNAi-based screen. RNAi lines to knock down each of the individual receptors were expressed in glia or neurons. The cross was conducted blinded, so that the identity of the receptors were not known until the data had been collected.

\begin{tabular}{|l|l|l|}
\hline RNAi line & Elav-RNAi & Repo-RNAi \\
\hline $5-H T 1 a$ & Normal & Normal \\
\hline $5-H T 1 b$ & Normal & Normal \\
\hline $5-H T 2 a$ & Normal & Normal \\
\hline $5-H T 2 b$ & Normal & Normal \\
\hline $5-H T 7$ & Normal & Failed to adapt \\
\hline
\end{tabular}

Table 4.1 - An RNAi-based screen revealed a dependence on the 5-HT7 receptor in glial cells for successful adaptation. All other receptors observed are unessential in neurons, and no other receptors are required in glia ("normal"). Failure to adapt was determined by the degree of asymmetric movement observed 240 minutes after the attachment of the splint.

In this screen, most genotypes adapted normally after 4 hours, as we observed in wild-type OR larvae. Two genotypes displayed a slightly weaker adaptation phenotype, which consisted of successful adaptation, but retained a 
slightly higher level of crawling asymmetry than was typically observed in wild-type larvae. However, the strongest phenotype observed was Repo>5-HT7 RNAi, in which the 5-HT7 receptor expression had been decreased in glial cells. The neuronal knockdown of the 5 -HT7 receptor adapted by 240 minutes post splint attachment as we had observed in OR larvae. This result is very interesting, largely because glial expression of 5-HT7 has been reported in mammalian vertebrates and glioblastoma cell lines (Mahe, 2004). Spinal cord 5-HT7 has also been implication in respiratory circuit plasticity, although it has not been determined whether the receptor is acting in neurons or glia in that context (Hoffman, 2011).

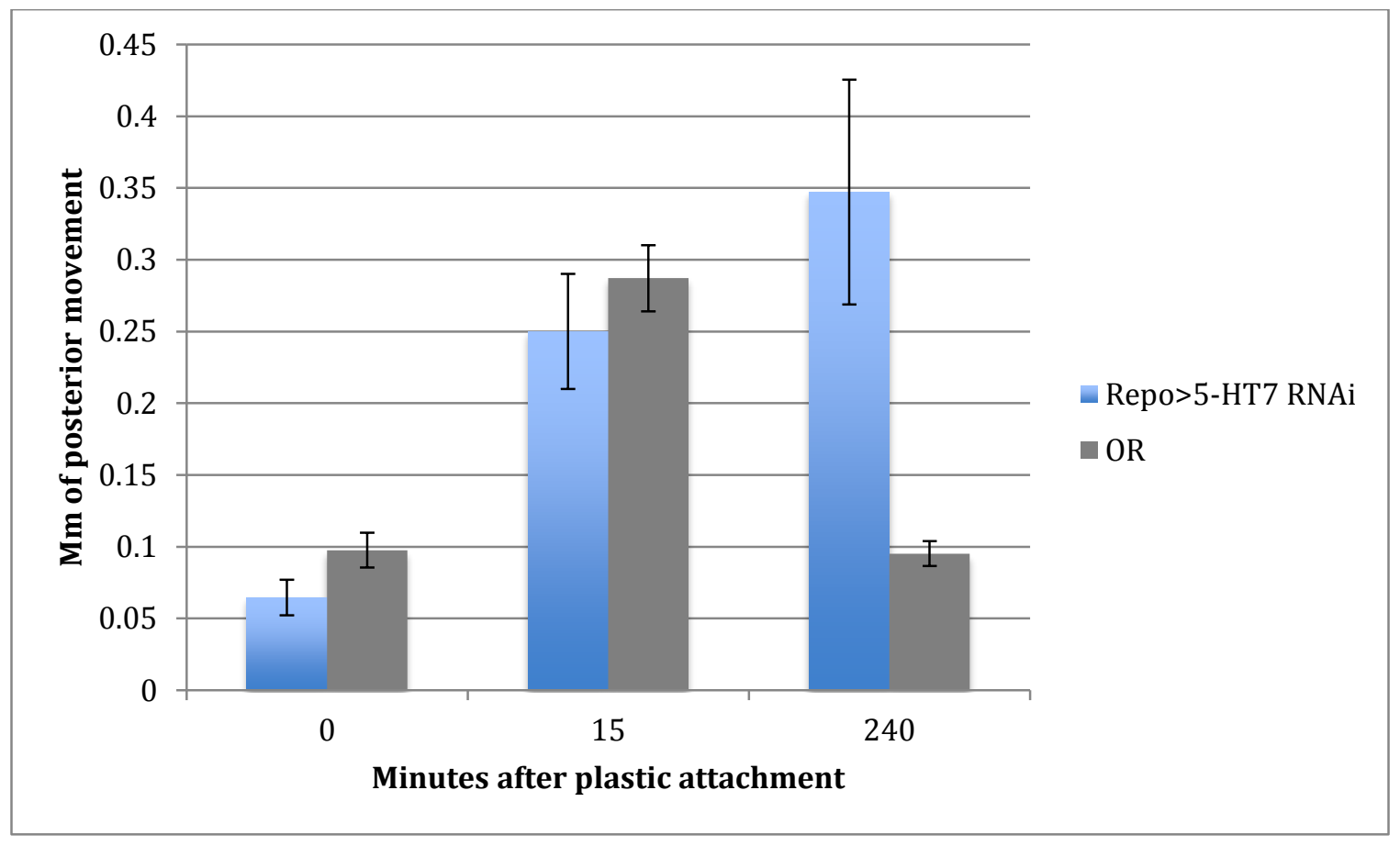

Fig. 4.4 - Glial specific knockdown of the 5-HT7 receptor prevents larvae from successfully adapting 4 hours after plastic attachment. When the 5-HT7 receptor was knocked down in glial cells (5-HT7), the asymmetric crawling pattern observed at 15 minutes after adaptation persisted up to 4 hours after adaptation (blue bars). Degree 
of asymmetry observed at 4 hours was statistically different from asymmetry observed in unsplinted larvae $(p<0.05)$, but not statistically different from the asymmetry observed at 15 minutes. Oregon $R$ larvae are shown for comparison (grey bars). $N=5$ for Repo-5HT7-RNAi, $N=8$ for OR controls.

\section{Additional results from the RNAi Screen}

Although the glial knockdown of 5-HT7 was the only positive result obtained in the entire screen, it is worth mentioning that several other genotypes displayed weaker phenotypes in the course of the locomotion assay. Time points were taken from all genotypes at 0,15 , and 240 minutes, and their adaptation was evaluated. One such result is the neuronal knockdown of 5-HT7 (elav>5-HT7 RNAi). In order for a genotype to be considered a positive hit in our screen, the degree of asymmetric crawling at 240 minutes must be statistically different than the time point at 0 minutes, and statistically the same as the time point at 15 minutes. Because the 240 time point was not statistically different from either the 0 or 15 minute time points, these larvae were considered to have an intermediate phenotype. One potential explaination of this, is that elav-gal4, a widely used neuronal marker, has been shown to have transient expression in neuroblasts and glial cells in fly embryos (Berger , 2007). Several cells within each segment during stage 12 exhibit cells which express both repo and elav. This result doesn't preclude the involvement of neuronal 5-HT7, however this is a possible complication worth noting. 


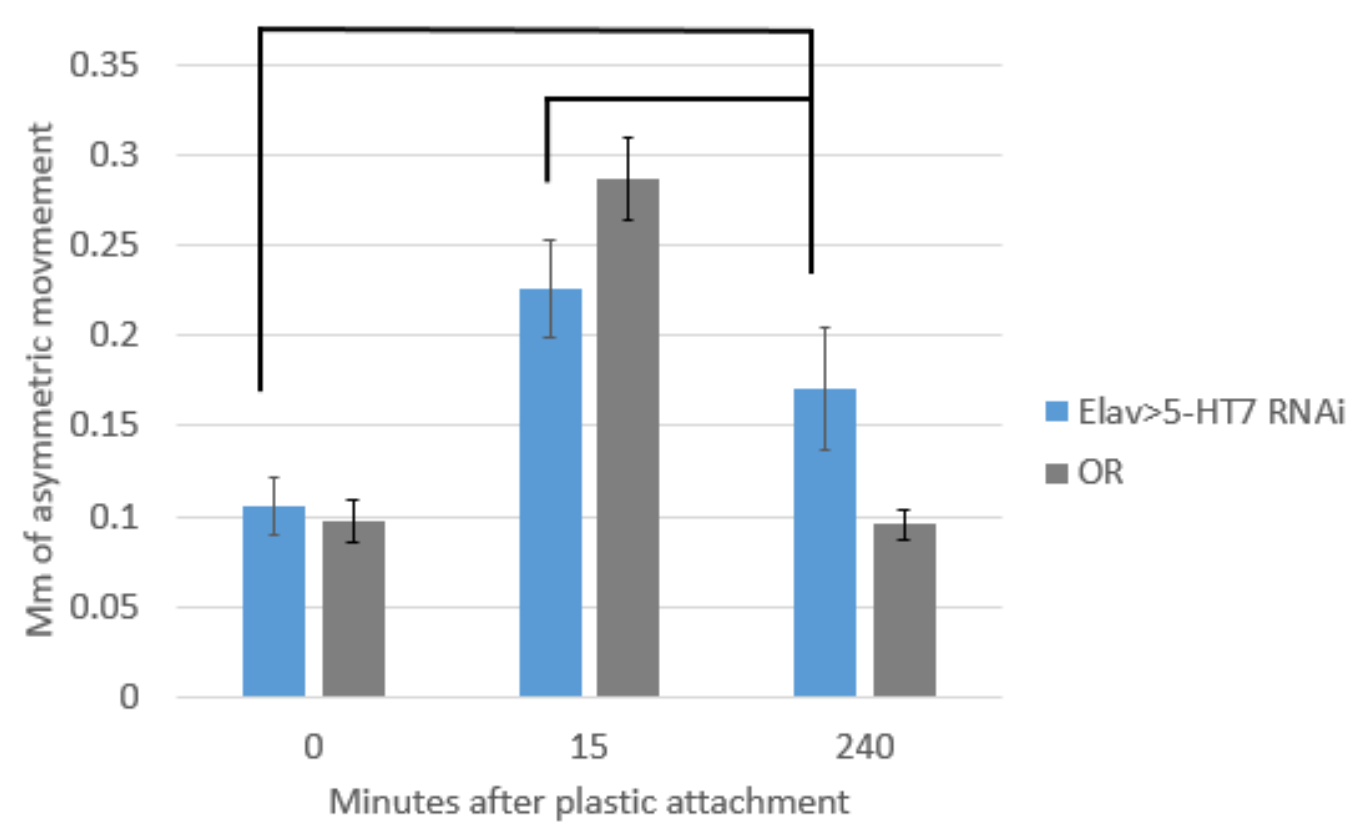

Fig. 4.5 - Reducing the expression of 5-HT7 in neurons results in insufficient adaptation. The neuronal knockdown of 5-HT7 (elav>5-HT7 RNAi, blue bars) develop the asymmetric crawling pattern after 15 minutes, but do not completely recover at 240 minutes. Grey bars indicate OR wild-type control for reference. $N=5$. Error bars indicate the non-significant difference between Elav>5-HT7 RNAi values at 240 minutes and any other time point. $N=4$ for Elav $>5-H T 7$ RNAi, $N=8$ for OR controls.

\section{Discussion}

We have previously demonstrated that the larval sensorimotor circuit is capable of adapting to the presence of an attached impediment during crawling. Next, we wanted to examine the role of a putative neuromodulator in the adaptation process. We chose to probe examine serotonin for several reasons. First of all, serotonin has been shown to modulate locomotor activity in both vertebrate and 
invertebrate systems. Secondly, several serotonin receptor types have been implicated in larval crawling. Finally, genetic tools are readily available to examine the requirement for other serotonergic signaling components in the adaptation process.

It is important to note that the results that when we increased expression of the serotonin transporter can cause more rapid clearance of serotonin at the synapse, effectively mimicking a decrease in serotonin levels. This is the primary mechanism of action by with antidepressants work. As we observed in our results with pCPA, decreasing serotonin causes delayed adaptation. With such a result, you would expect that overexpression of dSERT to mimic the phenotype observed by larvae treated with pCPA. However, we observed the opposite phenotype. While these data seem incompatible, one explanation for how these results work together is that increasing serotonin at the increased release in serotonin at the presynaptic membrane causes an increase in the serotonin released by these neurons. As a result, which is enough to overcome the rapid clearance associated with the additional transporter expression.

Increasing synaptic serotonin, through the addition of 5-HTP, overexpression of tryptophan hydroxylase, and through overexpression of dSERT on the serotonergic neurons, all resulted in rapid adaptation. In the case of dSERT, adaptation occurred more rapidly than in any other situation. This could be due to differences in serotonin available at the synapse in the $T r h>d S E R T$ larvae compared to the other two manipulations. Alternatively, there may be differences between these conditions with respect to where serotonin is localized in the cell. Increasing 
serotonin with overexpression of trh or with the addition of 5-HTP increases serotonin within the serotonergic neurons, however the overexpression of dSERT increases serotonin specifically at the pre-synaptic membrane on the serotonergic neurons. The especially rapid adaptation observed in the Trh>dSERT is likely due to the additional availability of serotonin at the synapse.

To gain an understanding of how serotonin is acting to facilitate adaptation, we decided to examine the role that serotonin receptors are playing in adaptation. Because serotonin receptors are present on glial cells and neurons, we wanted to examine the requirement of receptors in each cell type. We were able to utilize existing RNAi lines available to each receptor type, and drive them in either glia or neurons using the gal4/UAS system. We used repo-gal4, which drives expression in all glial cell types, and we used elav-gal4 which is a pan-neuronal driver. Each of these drivers were crossed with RNAi lines to the known serotonin receptors. Because the only genotype that failed to adapt was the Repo>5-HT7 RNAi line, we concluded that serotonin mediates adaptation exclusively through 5-HT7 receptors in glia. This is especially interesting, because although glial expression of 5-HT7 has been shown, and the role of spinal 5-HT7 has been demonstrated in sensorimotor plasticity, this is the first demonstration that glial 5-HT7 expression may be the necessary receptor and cell type to facilitate adaptation.

Mechanistically, it is still unknown how glial 5-HT7 could contribute to adaptation in this circuit. Activation of 5-HT7 on glia could elicit several different responses which could facilitate plasticity. 5-HT7 activates adenylyl cyclase (AC), resulting in an increase in cAMP (Ruat, 1993). The second messenger cAMP, in turn, 
could be influencing signaling cascades within the glia by either altering its morphology, its electrical properties, or initiating the phosphorylation of substrates downstream of PKA (reviewed in Taylor, 2004). In response to cAMP increase, glia could be releasing a signal to cause changes in a neuronal component of the adaptation process.

The results obtained by the overexpression of dSERT were dramatic, but not necessarily expected. Our hypothesis had been that an increase in dSERT would mimic a decrease in serotonin phenotype, due to the fact that serotonin levels observed in overexpression studies show a concentration of serotonin at the presynaptic terminals in the serotonergic neurons (Park, 2006). However, the phenotype we observed was that of adaptation so rapid that we initially did not catch it, as it happened before the first time point that we had normally collected, 5 minutes after attaching the plastic.

Although this was not the result that we had initially anticipated, it is informative about the action of serotonin in the facilitation of adaptation. These results indicate that high levels of serotonin specifically at the synapse result in rapid adaptation. One way that this could influence adaptation is by increasing the amount of serotonin released at the synapse with each action potential. This would need to be confirmed using cyclic voltammetry, a technique which has been used to measure the contribution of dSERT to replenishing the serotonin pool at the synapse (Borue , 2010).

Because this result is unexpected, it is important to consider additional potential explanations for this phenotype. One possibility is that, by overexpressing 
dSERT throughout all developmental stages, we are altering properties of the serotonergic neurons themselves. Disrupting serotonergic signaling has been shown to alter morphology and connectivity of serotonergic neurons (Daubert, 2010), so it is possible that we are causing a change in the larger network of serotonergic neurons. In order to examine this possibility, additional experimentation would need to be done. These questions could best be addressed with a combination of microscopy, and electrophysiology. Confocal microscopy, using GFP driven in the serotonergic neurons, could provide insight into the structure of serotonergic neurons. Any changes in structure might be visible when the membrane of the serotoninergic neurons are labeled. However, an additional marker might be required: a synaptic GFP (Syt-GFP). Syt-GFP would label the synapses of serotonergic neurons, whose quantities and location could be changing throughout adaptation.

Electrophysiology could confirm that the overexpression of dSERT has a phenotype on the activity of neurons in the sensorimotor circuit. Similarly to the experiments conducted by Fox and $\mathrm{Wu}$, recordings could be taken from the abdominal nerves, which contain the sensory and motor neurons (Fox and Wu, 2006). Because we our current model proposes that adaptation is occurring by changes in segment timing, we might observe altered timing of segmental nerves in the dSERT larvae.

The data presented here pertaining to serotonin levels, receptors, and cell types, provide insight into how serotonin impacts the connections between CPGs. In 
addition, the use of these genetic and pharmacological tools in conjunction with our behavioral assay demonstrated its application and versatility as a scientific tool. 
Chapter 5 - The sensorimotor adaptation phenotype is temperature sensitive. 


\section{Abstract}

Many of the most powerful tools in Drosophila neurobiology involve the use of temperature to temporally control their activity. Larvae can be exposed to heat at any time during an experiment, or any developmental time period, and the temperature exposure is reversible. Ectopic expression of $\operatorname{TrpA1}$, a temperature sensitive ion channel, or the temperature sensitive allele of shibire, the Drosophila gene expressing dynamin, are among the most common ways to increase or decrease neuronal activity, respectively. In the process of probing the role of individual neurons and glial cells in the adaptation assay described earlier, we exposed Oregon $\mathrm{R}$ larvae to elevated temperature as a control for future experiments with shibire. To our surprise, simply elevating the temperature while larvae was enough to reduce the asymmetric posterior movement observed at room temperature. When larvae were subsequently returned to room temperature, the asymmetric crawling pattern returned to a level comparable to the level observed prior to the temperature elevation. Although we do not currently understand how the adaptation phenotype is regulated by temperature, the fact that it is reversible suggests that we are not observing true adaptation with elevated temperature, but we are forcing the phenotype by activating a component normally activated late in the adaptation process. Although these results exclude the possibility of using temperature sensitive tools in this assay in the future, these results provide us with an additional understanding of the components involved in sensorimotor plasticity. 


\section{Introduction}

Drosophila larvae have very specific temperature preferences that correlate with desirable features of their environment. In the wild, larvae usually select for environments which are cool enough to prevent desiccation and heat damage, but warm enough to retain normal movement and physiological function.

How larvae sense temperature is controlled by many sensory neurons, and a variety of ion channels on these sensory neurons. Different neurons and different ion channels are activated in different temperatures (reviewed extensively in Dillon , 2011). Of particular interest, however, are the thermosensitive neurons located on the larval body wall. Many of the nociceptive neurons mentioned in previous chapters also express temperature-sensitive ion channels. These ion channels vary widely with respect to the temperatures that they detect, as well as the ranges of those temperatures. The most widely studied group of channels which influence temperature are transient receptor potential, or TRP channels (reviewed by Dhaka, 2006). The most widely studied with respect to temperature sensitivity in Drosophila is the TrpA1 channel (Rosenzweig , 2006). TrpA1 is a channel capable of responding to a range of stimuli, from moderate warming to noxious heat. Isoforms of the TrpA1 gene which are specific to neurons sensing noxious heat express an isoform of TrpA1 which is only activated by higher temperatures (Kang , 2012).

Other channels are involved in temperature sensitivity as well, including ANKTM1, which is sensitive to very cold temperatures, down to about $11^{\circ} \mathrm{C}$ (Story, 2003). Another TRP channel, TrpM8, is sensitive to menthol as well as mild cool 
temperatures $\left(<25^{\circ} \mathrm{C}\right)$ (McKemy, 2002). Interestingly, these TRPA1 and TRPM8 proteins, when driven by a UAS sequence are able to be used as tools to confer temperature sensitivity on groups of neurons as a means to manipulate their activity (Berni, 2010; Peabody, 2009). Rhodopsin a protein crucial for vision in adult and larval flies, is also known to be expressed in the larval body wall where it mediates sensitivity to temperature (Shen, 2011).

An interesting study by Liu showed, using an intracellular calcium indicator as a measure of activity that many of the sensory neurons in the larval body wall are capable of responding to changes in temperature (2003). Interestingly, almost every group of neurons expressed a unique temperature sensitivity profile (Liu, 2003). While this is an interesting observation pertaining to the nature of larval sensory neurons, it also has profound implications for the study of neural circuits. Many of the tools used to study neural circuits are temperature sensitive, including TRPA1, TRPM8, and shibire ${ }^{T S}$. The research published by Liu suggest that more attention may been to be paid to the potential confounding results that these neurons may produce.

Temperature tolerance and locomotion in wild Drosophila is shaped heavily by geographic region, population, and life history (Boher, 2010). Although it is understood that this thermal preference correlates with preferences in digging behavior, it is not well understood how temperature controls patterns of locomotion and changes in locomotor behavior. In most experiments where a temperature sensitive tool is used, the wild-type larvae also display a slight increase in the rate of locomotion in response to increased temperature (up to an increase of about $5^{\circ} \mathrm{C}$ ), 
and show a gradual slowing down to totally arrested locomotion as the temperature decreases.

In an attempt to identify sensory neurons which may be involved in adaptation in our locomotion assay, we employed the use of shibire ${ }^{T S}$ in order to manipulate the activity of specific groups of sensory neurons. In addition, we had planned on trying to recapitulate our loss of serotonin experiments by silencing the serotonergic neurons with the same tool. However, in the course of these experiments we found that temperature itself had a profound impact on performance in our locomotion assay. When larvae were shifted to a higher temperature, the asymmetric crawling pattern decreased, giving the appearance of adaptation. However, when these larvae were returned to room temperature, they resumed crawling with the asymmetric pattern that they had displayed prior to the temperature shift. We were able to place these larvae in the increased temperature again, and were able to see that result a second time as well, in the same larvae. These results indicate that higher temperatures are capable of reversing the asymmetric crawling phenotype, without actually resulting in adaptation.

\section{Results}

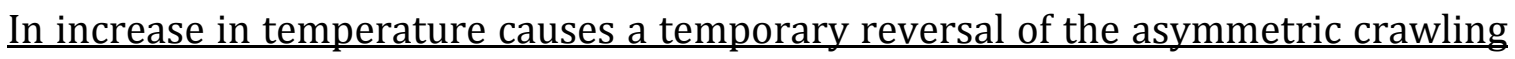
phenotype.

In the process of performing a control experiment, we placed OR larvae in an incubator set at 30C. When we recorded videos of larvae that had been kept at 30C for 15 minutes, their asymmetric crawling pattern had very significantly diminished 
(Fig. 5.1). When these same larvae were observed at room temperature prior to being heated. One possible explanation for this phenotype is that the increased temperature increased the adaptation phenotype, possibly due to increased locomotion of the larvae. To test this, we returned the larvae to room temperature, and allowed them to crawl for 15 minutes before observing their crawling pattern. Surprisingly, after the larvae had been at room temperature they resumed the asymmetric posterior movement that we observed prior to their transfer to the elevated temperature (Fig. 5.1). These data indicate that there is a temperature sensitive component to the adaptation phenotype, which is distinct from the adaptation process itself.

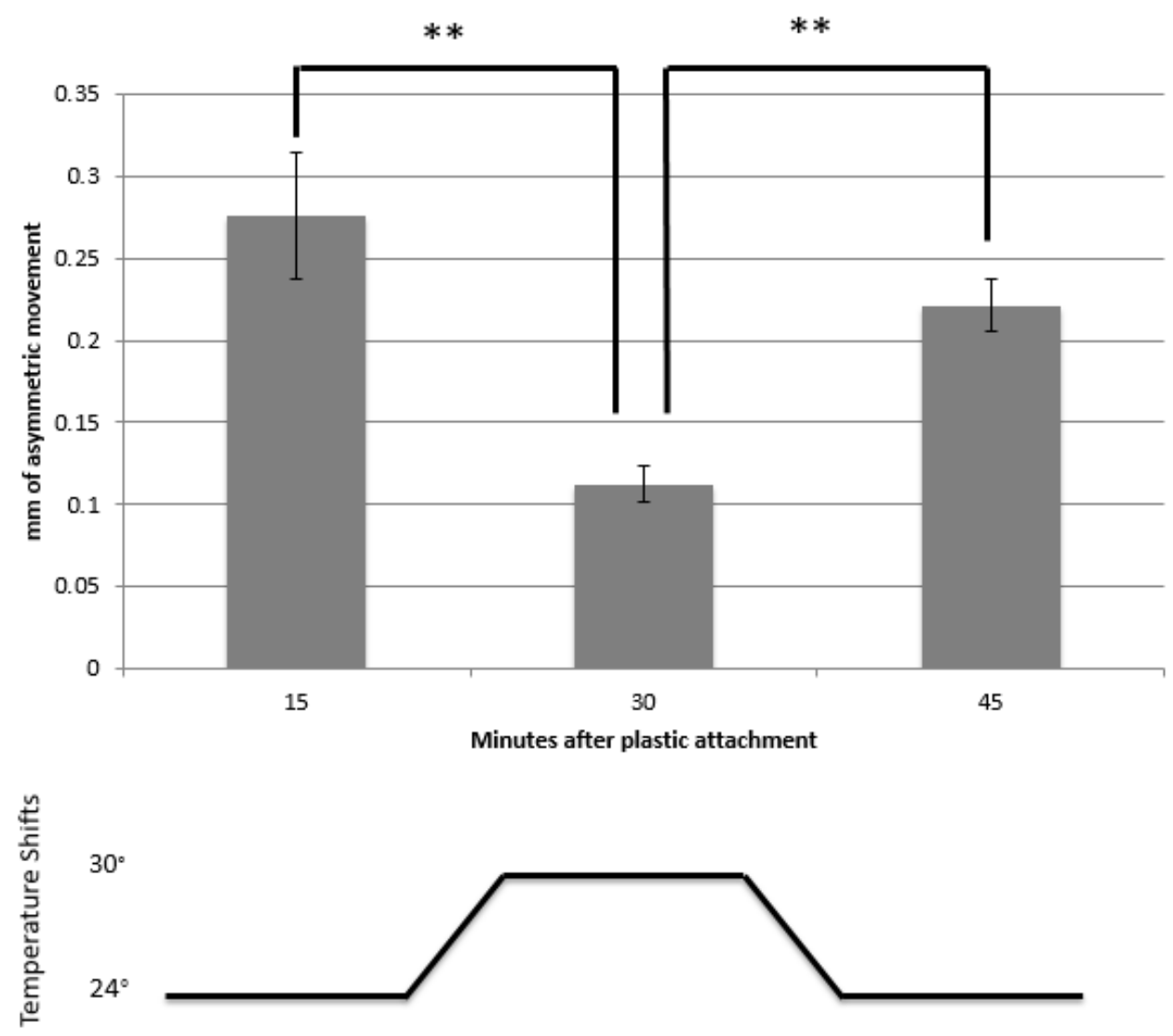


Fig. 5.1 - The asymmetric posterior movement transiently disappears at elevated temperatures. OR larvae were splinted, and then placed at room temperature for 15 minutes. After data were collected, larvae were moved to an incubator at 30degrees Celsius for 15 minutes. Data were collected, and then larvae were returned to room temperature for 15 more minutes (temperature shift scheme indicated below). $p<0.01, N=5$.

\section{Discussion}

Temperature is a widely-used tool for the manipulation of neurons and genes in Drosophila. Tools like shibire ${ }^{T S}$, temperature-sensitive gal80 (gal80 ${ }^{\mathrm{TS}}$ ), and ectopic expression of the $\operatorname{Trp} A 1$ gene are all tools used which utilize temperature shifts in order to act. Because we see a phenotype in our assay that is independent of the use of any of these tools, we are unable to use temperature sensitive tools in our assay without observing confounding effects.

These results indicate that some component of this adaptation phenotype that we observe is temperature sensitive. The nature of this temperature sensitivity is unclear, but I believe that a good candidate would be in the temperature sensitive neurons in the body wall. Many of the neurons in the body wall of Drosophila larvae are sensitive to different temperatures (Liu, 2003). Because this assay is disrupting the CPGs' expected sensory feedback, activation of sensory neurons may influence the asymmetric phenotype. 
In order to further address the temperature sensitivity, we will be very limited as to the tools that we are able to use to identify the neurons and genes involved. However, mutants are available for most of the TRP channels that are expressed in these neurons. In addition, RNAi directed to specific groups of sensory neurons could tell us more about the role of sensory neurons in adaptation. By manipulation of the expression or activity of the temperature sensitive channels in specific neurons, we hope to gain an understanding of how temperature impacts the neurons within and between the CPGs controlling locomotion.

The impact of temperature, to be sure, provides an inconvenient limitation to the tools available in this assay. However, this provides a very valuable insight into a potential pitfall of the use of shibire ${ }^{T S}$ in other larval locomotion studies. If temperature has an influence on locomotor behavior, it is something that should be taken into account. Earlier I mentioned a paper published by Hughes and Thomas, in which they used shiTs to inhibit the activity of specific groups of sensory neurons (2007). While they also used a genetic ablation technique in conjunction with their temperature-driven experiments, our results suggest that there might be a benefit to confirming their results with a non-temperature sensitive mechanism. For example, their results could be very easily replicated using channelrhodopsin (ChR) to activate neurons, or halorhodopsin (HR) to silence neurons. These results do not invalidate the results that Hughes and Thomas published, but it does suggest that there is another level to their results.

An additional consideration for the temperature sensitive phenotype is that what we observe here may not be a phenotype of the sensory neurons. One 
possibility is that we are changing the texture of the substrate that the larvae are crawling on. Indeed, the shift in and out of the incubator could cause condensation to form on the surface of the agar plate. The best way to test this possibility directly would be to conduct this experiment on several different surfaces, each with varying concentrations of agar, and various amounts of water on the surface of the agar. If significant differences in amount of segment asymmetry are observed, we can reasonable conclude that surface characteristics have an influence on the adaptation phenotype that we observe. 
Chapter 6 - Discussion, future directions, and broader impacts 


\section{Summary}

Central pattern generators (CPGs) are the basis for rhythmic neurological functions (Harris-Warrick, 2010). Using a limited set of components, CPGs are able to govern breathing, locomotion, and digestion. Drosophila larvae use a series of repeating CPGs to crawl, with each CPG responsible for an individual segment. It isn't clear, from the literature, exactly how these CPGs communicate or respond to feedback from one another. To address this, we developed a locomotion assay in which we immobilized two of the posterior-most segments with a plastic splint. Larvae with this splint were still able to crawl, but crawled with an exaggerated asymmetric movement of the posterior end caused by disruption in the timing of compression of the segments surrounding the splint. After approximately four hours, however, the larvae had adapted to the presence of the splint and had reduced the asymmetric crawling pattern. If the splint was subsequently removed, we observed that the asymmetric crawling pattern would return for a short period of time, before reverting back to crawling straight.

Because CPGs are highly regulated, both by their own activity and by external neuromodulators, we wanted to find out how sensorimotor adaptation is regulated in larvae. Serotonin is a neurotransmitter with an established role in regulating locomotion and neural circuits in both vertebrates and invertebrates, which makes it a very strong candidate for regulating the plasticity observed in our assay (HarrisWarrick, 2010). Indeed, when we inhibit serotonin synthesis, adaptation fails to 
occur after four hours, contrary to what we observed in wild-type larvae. When we increase serotonin synthesis, adaptation occurs significantly faster than observed in wild-type larvae. From this, we can infer that serotonin is required for adaptation to occur, and can instruct adaptation when serotonin synthesis is increased. Another component of signaling in serotonergic neurons which we probed is the serotonin transporter, SERT. We overexpressed the Drosophila serotonin transporter (dSERT) in serotonergic neurons to increase the amount of serotonin present at the synapse. Interestingly, these larvae developed the asymmetric crawling pattern incredibly rapidly (by 5 minutes after the attachment of the splint) and had completely adapted by 15 minutes after the attachment of the splint. This result is contradictory to what we would expect, given that an increase in SERT activity decreases the serotonin present in the synapse. This suggests that the availability of serotonin at the pre-synaptic membrane of serotonergic neurons is the primary influence on adaptation.

Serotonin acts through one of 4 receptors in Drosophila - 5-HT1a, 5-HT1b, 5HT2, or 5-HT7. To increase our understanding of the role of serotonin in adaptation, we directed an RNAi-based screen, knocking down each receptor in either glia or neurons, and looking for larvae that were unable to adapt. Larvae in which the 5HT7 receptor had been knocked down in glial cells (Repo>5-HT7 RNAi) were the only genotype which failed to adapt. All other receptors on glia or neurons were not required for successful adaptation in larvae.

Finally, we have evidence to suggest that there is a temperature sensitive component within the adaptation process. When Oregon R (OR) larvae are placed in 
a higher temperature environment, the asymmetric crawling pattern disappears, and larvae appear to have adapted. However, when these larvae are moved back to room temperature, the asymmetric crawling pattern returns. In other words, larvae do not adapt at the higher temperature, but higher temperatures are capable of masking the asymmetric phenotype. Although it is not entirely clear which component of the circuit is being affected by temperature, the most likely candidate is that the temperature shift may be causing an effect in one of the temperature sensitive sensory neurons in the larval body wall.

In this dissertation I have described a novel assay, and have proven its use in examining the role of serotonin and temperature in sensorimotor plasticity. This assay can be used in conjunction with any non-temperature-sensitive genetic tools or pharmacological treatments to address the components of sensorimotor plasticity.

Serotonin regulates adaptation in the larval sensorimotor circuit

Serotonin has been shown to influence plasticity in a large number of neural circuits. Respiration, locomotion, and digestion all have patterns of activity that are regulated in some capacity by serotonin and its receptors. Misexpression or misregualtion of serotonergic signaling components results in malfunction in each of these biological processes (reviewed in Daubert and Condron, 2011). In addition, serotonin in the subesophageal zone (which contains another CPG) increases the activity of motor neurons in the esophagus which contribute to the movement of food through the digestive track (Schoofs , 2014). In addition, very recent work by Albin (2015) demonstrates a role of serotonin in behaviors associated with satiety 
and hunger, including locomotion (Albin , 2015). This adds a potentially interesting aspect to our results, as it is possible that serotonin may be causing adaptation through descending serotonergic input, or by acting locally within the CPG. Currently, our assay cannot distinguish between the two. However, this question could potentially be addressed through the use of tsh-gal80, which excludes expression in a gal4 UAS system in the brain. Although it is possible that descending control from the brain is involved, I hypothesize that further research will reveal that serotonin is acting at or near the segment of the ventral nerve cord (VNC) where the splint is located. The serotonergic neurons exist on the left and right of each abdominal segment, and send projections to the opposite half of the segment (Valles and White, 1988). Because a given serotonergic neuron is capable of communicating with the contralateral side, they are a very good candidate for neurons which could coordinate timing between the left and right of each segment.

Because increasing serotonin caused rapid adaptation, we had hypothesized that we could observe an increase in serotonin in the VNC where adaptation was primarily occurring. A caveat to this experiment is that we were unable to observe immunohistochemical changes in serotonergic neurons in larvae before and after adaptation. Quite a bit of time was spent trying to observe changes in serotonin levels between the left and right serotonergic neurons in the VNC. However, we were unable to observe any obvious changes in the quantity of serotonin between these groups of neurons, nor were we able to see any consistent changes in serotonergic neuron structure. 
The 5-HT7 receptor could potentially have a multifaceted role in adaptation

The 5-HT7 receptor has been implicated in the plasticity of several sensorimotor circuits. Phrenic motor facilitation, a breathing pattern induced by acute intermittent hypoxia, is dependent upon expression of the 5-HT7 receptor in the cervical spinal cord (Hoffman, 2011). Stimulating the 5-HT7 receptor in the cervical spinal cord of rats is sufficient to increase the tidal volume during inhalation. When this receptor is knocked down using RNAi, phrenic motor facilitation does not occur, even in the presence of hypoxic conditions. In this circuit, researchers demonstrated that a very specific physiological change occurred simply through manipulation of the 5-HT7 receptor. In other words, 5-HT7 is important for interpreting necessary sensory input to cause a motor output change downstream. The 5-HT7 receptor in sensorimotor adaptation appears to carry out a very similar role, which is to allow the body segments surrounding the splint to compensate by adjusting their output.

In Drosophila, the role of the 5-HT7 receptor has been studied fairly recently in the context of mating and courtship behaviors in adult (Becnel, 2011). Although this study revealed the role of serotonin in these behavior in the adult, Becnel and colleagues also were among the first to characterize the expression of the 5-HT7 receptor in both larval and adult central nervous systems. Although their primary interest was in the neurons of the central complex, they were able to use the 5-HT7 promoter region to identify expression in interneurons and apparently glial subtypes in the ventral nerve cord. These cells occur in a segmental pattern in the ventral nerve cord, which is compatible with a putative role for them in larval 
locomotion (Becnel, 2011). What remains unclear, however is how 5-HT7 expression in glia cause adaptation to occur in larvae.

To truly gain an understanding of how 5-HT7 acts, we need to observe the location of 5-HT7 on the target cells. A major inconvenience which prevents us from actually observing this is that there is no antibody that exists to label the 5-HT7 receptor. One potential way around this, would be to make a GFP-tagged version of the 5-HT7 receptor. In larvae with this transgene, we would be able to confirm where the 5-HT7 receptor is located, and we would be able to observe whether this receptor changed over the course of adaptation with respect to amount or location. A 5-HT7-gal4 transgenic fly exists, driving the gal4 gene with the 5-HT7 promoter (Becnel, 2011). However, this tool does not allow for the observation of the actual receptor protein in vivo.

Glia play an active role in sensorimotor circuit activity

The importance of glia in the development, maintenance, and function of the nervous system is becoming increasingly appreciated. In the components of the sensorimotor circuit especially, glia are associated with every neuronal element (Santello, 2012). A particularly interesting example in the literature is that of the sensory organ near the amphid of $C$. elegans. This organ contains several neurons that are ensheathed in glia. When these glia are ablated, the C. elegans exhibit a number of difficulties with respect to neuronal morphology, as well as defects in thermotaxis behavior mediated by these neurons (Bacaj, 2008). This study is especially profound, because it highlights the impact of glia not only on the health of those neurons, but the impact of specifically the glia on a stereotyped behavior in $C$. 
elegans. Furthermore, Bacaj and colleagues were able to identify a specific protein, FIG-1, a secreted thrombospondin, which acts extracellularly and is required for the execution of thermotaxis (Bacaj, 2008).

Thrombospondins are proteins secreted by glia which elicit synaptic remodeling in neurons (Christopherson, 2005). Interestingly, glial-secreted thrombospondins have been shown to stabilize the innervation of motor neurons which control flight in adult flies, (Hebbar and Fernandes, 2010). Hebbar and Fernandes report that the crucial point of contact between glia and motor neurons occur in the muscles controlling flight, where they directly cause changes in synaptic connections (Hebbar and Fernandes, 2010). Although thrombospondins in this circuit are acting in response to a developmental cue over the course of a much longer time period, this is probably the best candidate for a means by which glia could be mediating plasticity in this circuit.

Again, one of the major limitations in our understanding of glia's mechanism of action is that we do not currently know how glia are eliciting changes that bring about sensorimotor adaptation. We do not know for certain whether a synaptic change is happening in an interneuron such as the evenskipped+ interneurons, or the leucokinin-expressing neurons which are implicated in asymmetric compression, or whether a change could be happening in the proprioceptors.

Although I have not discussed it in detail, another possible component for adaptation is a change in the motor neurons. Motor neurons, like sensory and interneurons, exist in repeating units in each segment, each controlling a group of nearly identical muscles (Heckscher, 2012). Different groups of motor neurons 
compress to cause forward and reverse crawling (Hecksher, 2012), or turning (Lahiri, 2011). In addition, serotonin can alter the electrical properties of larval motor neurons (Dasari and Cooper, 2003). In other words, motor neurons could be modified as a means to bring about sensorimotor adaptation. As I mentioned earlier, serotonin is unlikely to be acting directly on the neuron itself, due to the results observed in the 5-HT7 knockdown in glia. However, glia could be bringing about changes in the motor neurons through a secreted factor.

Temperature could regulate adaptation through sensory neurons, or by modifying the properties of the crawling substrate

One of the most fascinating, yet most frustrating, results obtained in experiments was the realization that the plasticity phenotype had some dependence on temperature. This is interesting, as there are a number of temperature sensitive sensory neurons which could be contributing to this phenotype, however, it also severely limits the tools available to use to probe the involvement of specific neurons in the adaptation process. Virtually all of the current methods for addressing individual neurons within a circuit involve either optogenetic or temperature-mediated control. Light activates several groups of neurons on the body wall, some of which are mechanosensitive. Using blue light to activate neurons with channelrhodopsin could potentially cause confounding results by activating these neurons in addition to those that we would be trying to manipulate genetically.

An alternative explanation, is that temperature is influencing the actual physical mechanics of larvae crawling on the surface of the plate. The agar substrate 
that the larvae are crawling around contains a large amount of moisture, which could accumulate condensation as the plate is moved from one temperature to another. Additional fluid could potentially have a large impact on the grip that the larvae have on the agar, which would understandable impact the crawling dynamics. One of the most interesting larval crawling phenotypes in the literature, nocifensive writing behavior, has received a lot of criticism in the literature because it only occurs on agar with a large amount of water on the surface (Hwang, 2007). Substrate moisture is a potentially important confounding variable, which could be easily tested. Making several batches of molasses agar with varying concentrations of agar would make be one way to address this question. In addition, a small amount of water could be added to the surface of the agar. If these conditions show variable results, it could indicate that the properties of the agar surface could contribute to the changes in adaptation observed in the temperature shift experiments.

\section{Future Directions}

The research presented in this dissertation describes an assay for sensorimotor plasticity using Drosophila larvae. We have demonstrated the requirement of serotonin and the 5-HT7 receptor on glial cells in the adaptation process. The description of this assay, the role of serotonin in sensorimotor plasticity, and the involvement of the 5-HT7 receptor and glial cells are all novel contributions to our knowledge of sensorimotor circuits. In addition, this research probes into the relationship between connected CPGs in a common function, which is not currently well understood. Although we have identified a behavioral consequence of the connection between larval CPGs, understanding the physical 
changes in connectivity and the specific cells and signaling molecules involved are still required for a complete understading.

The sensorimotor plasticity assay described here can be used in conjunction with genetic and pharmacological tools to examine the role of different neurotransmitters and signaling components in adaptation. Here, we have used both to examine components of serotonergic signaling. However, there is a need for further research in determining more specifically which cell types are involved in the adaptation process. Although we know that glial cells are involved, through the use of more specific drivers we could potentially narrow down the specific location and nature of the glial cells involved in adaptation. In addition, tools such as channelrhodopsin and halorhodopsin could be used to modify the function of interneurons involved in the basic circuit. A large number of gal4 drivers are available to manipulate very specific groups of neurons, and in some cases single neurons. Knocking out individual neurons in larvae in this assay could provide us with the ability to determine which specific neurons are involved in adaptation.

As mentioned earlier, there currently exists no ideal way to manipulate neurons targeted to a specific abdominal segment in the larval periphery or central nervous system. Neuronal drivers exist to specifically activate virtually any subset of neurons of interest, even single neurons within each segment. However, it is virtually impossible to manipulate a single neuron within a single segment. One technique which could potentially be used for this kind of targeting is mosaic analysis with a repressible cell marker (MARCM). This is a widely used technique which can generate single cells with distinct genotypes from their neighboring cells 
(Lee and Luo, 2001). These clones can then be used to determine the contribution of single cells to a given phenotype. The only potential pitfall with the use of MARCM in this assay, is that the likelihood of positively marking a specific neuron in the same specific segment in each larva used in this assay is incredibly low. It would be very difficult to achieve significant results.

Another technique which may prove to be promising, is the use of the split gal4 system. This is a variation on the gal4/UAS system described earlier, except that the gal4 protein is split into two halves, and expressed using two distinct drivers. In other words, the first half of the gal4 may be expressed with a driver specific to motor neurons (OK371-lexA, for example), and the second half of the gal4 may be expressed in specific abdominal hemisegment (potentially using a combination of hox genes). This would ensure activation only of motor neurons, and only within the segment or segments of interest (Ting, 2011). However, this is once again dependent upon developing a means to drive expression focused on specific segments, which does not currently exist.

What are the connections between CPGs in the VNC?

One of the major questions which remain is "what are the nature of the changes in neural circuits during the adaptation process?" The mechanism proposed in chapter one requires the growth of a novel connection, or the strengthening of an existing connection. However, our results do not point strongly to one or the other. In order to determine how the connections between the CPGs are changing, we would need to narrow down where the crucial connections are prior to the addition of the splint. A candidate neuron in this case, would be one which influences the 
timing of motor neuron firing relative to another segment. A promising candidate group of interneurons include the period-expressing medial segmental interneurons (PMSIs), which fire just prior to relaxing of the motor neurons in a specific segment (Kohsaka, 2014). Very recently, an additional group of neurons were identified which synapse onto the motor neurons directly in each segment and silence their activity, glutamatergic ventro-lateral interneurons (GLVIs) (Itakura, 2015). What is interesting about these neurons, is that each segment contains one pair of them (left and right) and they have projections which form synapses on the dorsal side of both the left and right halves of the VNC. Not only do they synapse onto the motor neurons in their own segment, but they synapse onto the contralateral motor neuron as well. In the model proposed in chapter 3, we hypothesized that a connection was diminished (A6R to A5R), and a novel connection was activated across the VNC to the opposite side (A6L to A5R). By looking for morphological changes in these neurons and manipulating their activity, we could examine their role in the adaptation process.

Very recently, a paper was published which very thoroughly addressed questions proposed in chapter 3 , and several of the proposed experiments described in my future directions. Heckscher and colleagues identified a group of interneurons which are positive for the gene Evenskipped, which are responsible for left-right coordination between the muscle groups in an individual segment (Heckscher, 2015). Ablation of these neurons causes asymmetry with respect to the amount of compression (how much the segment contracts with each peristaltic wave). These neurons also receive synaptic input directly from proprioceptors and 
other sensory neurons, and are removed from motor neurons by one excitatory interneuron. These interneurons very well could be the interneurons that are being disrupted in our locomotion assay (Heckscher, 2015).

This paper utilized genetic and microscopy methods to describe the impact of these neurons on segment symmetry during crawling. Without these neurons symmetry is lost. This is slightly different from the assay described here, where we are inducing the segment asymmetry. However, many of the features of these neurons make them likely important for sensorimotor adaptation as well. For example, the interneurons receive synaptic input from their own proprioceptors, as well as proprioceptors from neighboring segments. In addition, they send projections across the midline in the VNC to the opposite segment. In other words, this neuron has many of the connections that we hypothesized an ideal candidate neuron would in chapter 3.

Now that evenskipped+ neurons have been identified, as well as several of their inputs and outputs, we can explore the role of these neurons in adaptation. The fact that segment symmetry can be disrupted by either activation or inhibition of evenskipped+ interneurons suggests that they function in a compensatory mechanism. In other words, they compare left and right symmetry and make adjustments accordingly. In response to prolonged asymmetry, synaptic remodeling might occur within this neuron to compensate for the induced asymmetry.

Chapter 4 describes the role of serotonin in gating the rate of adaptation to the splinted segment. From the information gathered in this paper, it is unclear how serotonin could be interacting with this neuron. The authors make no mention of 
serotonergic neurons interacting either upstream or downstream of evenskipped+ neurons. However, these interneurons could be made more or less susceptible to plasticity downstream of glia (discussed in greater detail above) (Heckscher, 2015). Similarities between turning behavior and induced segment asymmetry

Although it is definitely possible that the evenskipped+ neurons are involved in righting induced asymmetry, there are several additional candidates for neurons which could be crucial for adaptation. Neurons that are required for turning behavior could play a role in adaptation as well. Turning during forward crawling is characterized by a very stereotypical set of movements, and involves deliberate asymmetric compression of abdominal segments (Kohsaka, 2014; Lahiri, 2011). The asymmetric compression of abdominal segments requires functioning chorodotonal (cho) neurons (Caldwell, 2003), and in addition is requires leucokinin-expressing interneurons in the VNC (Kohsaka, 2014). What is of particular interest to this model, is that serotonin modifies the activity of these leucokinin-expressing neurons (Kohsaka, 2014). Because we identified the 5-HT7 receptor on glia, it is unlikely that serotonin's mechanism of action in adaptation is identical to the mechanism responsible for turning. However, there could be components in common between the two pathways. For example, glia could be acting on leucokinin-expressing neurons downstream of activation by serotonin. This could be parsed out by identifying the factor secreted by glia, and then observing an impact on leucokininexpressing neurons. The outcomes that we could look for would be changes in synapse number or changes in neurotransmitter level (either leucokinin or acetylcholine). Immunohistochemistry could be used to observe the changes in 
either quantity or location of the leucokinin in these cells, between unsplinted larvae, and splinted larvae.

Beyond Fruit Flies - what can be learned from model systems, and potential hope for clinical relevance.

As mentioned earlier, the basic organization of the central pattern generators controlling locomotion are conserved in invertebrates and vertebrates. In the central nervous system, interneurons generate oscillating patterns of activity which drive the behavior of motor neurons. Sensory neurons in the periphery regulate the activity of the neurons within the central nervous system. In cats, zebrafish, leeches, and Drosophila, serotonin is understood to play a role in locomotion. With so many conserved elements across these phylogenetic groups, it is likely that serotonin is playing a role in plasticity and adaptation of the sensorimotor circuits of other organisms as well.

In zebrafish, serotonin is important for the development of functional sensorimotor circuits (Brustein, 2003), and regulates fictive locomotion in preparations of the adult spinal cord (Gabriel, 2009). Although zebrafish swim through bending their bodies, rather than sequential compression of individual abdominal segments as Drosophila larvae do, zebrafish could provide interesting insight to the role of serotonin in CPG coordination and synchronization. Because locomotion is carried out in a slightly different way, it would be fascinating to see if serotonin could influence segment coordination in this system as well. In addition, the impact of serotonin on individual zebrafish could be observed. Because drugs 
can be fed in the water of fish being tested, this would provide a very compelling argument for a cause and effect of serotonin.

Although it would be very interesting and relatively simple to address the requirement of the 5-HT7 receptor for adaptation in other organisms, it would be very intriguing to investigate if it has a therapeutic application in human locomotion. Nerve damage, muscle atrophy, and other physical limitations can result in disruptive patterns of locomotion in recovering patients. Agonists and antagonists exist for all serotonin receptors and the serotonin transporter, and virtually all of them are currently used as a treatment for various cardiovascular, psychological, or digestive disorders. There are very few studies which examine the use of serotonin in physical therapy applications, but one study published in 1996 examined the impact that treatment with fluoxetine had on stroke victims receiving therapy for hemiplegia, or paralysis on one half of the body. Their results indicated that, compared with placebo, fluoxetine-treated patients performed better in daily activities, and regained their ability to walk more quickly (Dam , 1996). With the exception of one follow-up study, in which they administered fluoxetine closer to the stroke (within 5 days), very few studies examine the effect of fluoxetine on motor recovery (Chollet , 2011). Rather than treatment with fluoxetine, perhaps the understanding of the 5-HT7 receptor could be examined more closely in these patients. This would allow for more focused treatment, negating the side effects associated with usual treatment with SSRIs. Of course, this would need to come after gaining an understanding of 5-HT7 in vertebrate locomotor plasticity, however this result is certainly worth exploring. 
In conclusion, there remains a large body of work to be done regarding the nature of sensorimotor adaptation. However, we have demonstrated a novel way to test sensorimotor plasticity, and have uncovered several underlying components which regulate the adaptation process. 


\section{Appendix - Terms and Abbreviations}

5-HT - Serotonin, 5-hydroxytryptamine

5-HT7 - The 5-HT7 serotonin receptor

ChR - Channelrhodopsin, a light sensitive ion channel used to activate neurons with blue light.

CNS - Central nervous system, in Drosophila it is comprised of the brain and the ventral nerve cord (VNC).

dbd - Dorsal bipolar dendritic neuron. A multidendritic neuron which is thought to act as a proprioceptor, as described in Hughes and Thomas, 2007. Thought to behave similarly to the SRO neuron in Manduca sexta larvae.

Elav - a marker used to drive expression in neurons. Used in our RNAi based screen to knock down expression in neurons.

Gal4/UAS System - A system for ectopically expressing a gene in a specific cell type or at a specific developmental time. The transcription regulatory sequence attached to the gal4 determines where the gene is expressed. The gene expressed downstream of the UAS will then be expressed as dictated by the gal4-driver.

Gal80 - a protein which blocks transcription of genes downstream of UAS sequences. 
GVLI - glutamatergic ventro-lateral interneurons. Neurons which synapse directly onto the motor neurons, and inhibit their activity a period of time after that segment has fired (Itakura , 2015).

HR - Halorhodopsin, a light-sensitive ion channel used to silence neurons using yellow light.

MARCM - Mosaic analysis with a repressible cell marker. This technique developed by Lee and Luo (2001) is used to specifically manipulate one, or a small number of cells to examine the isolated phenotype

MD - Multidendritic neurons. A group of sensory neurons in the body wall, which possess the ability to sense temperature, light, and various types of mechanical stress.

PMSI - period-positive medial segmental interneurons. Identified by Kohsaka (2014) as neurons which are involved in silencing the activity of motor neurons after a segment has compressed.

Repo - A gene expressed specifically in glial cells, used in the form of repo-gal4 to drive glial expression in our RNAi screen.

Shi ${ }^{\mathrm{TS}}$ - A temperature sensitive allele of shibire, the Drosophila dynamin gene, which temporarily silences neurons at higher temperatures

VNC - Ventral Nerve Cord, the spinal cord equivalent of Drosophila larvae. 


\section{Works Cited}

Andersen, J. M., K. S. Sugerman, J. R. Lockhart, W. A. Weinberg (1997). Effective prophylactic therapy for cyclic vomiting syndrome in children using amitriptyline or cyproheptadine. Pediatrics 100(6):977-981.

Bacaj, T., M. Tevlin, Y. Lu, S. Shaham (2008). Glia are essential for sensory organ function in C. elegans 322(5902):744-747.

Bargmann, C. I. (2012). Beyond the connectome: how neuromodulators shape neural circuits. Bioessays 34:458-465.

Beasley, C. M., B. N. Masica, J. H. Potvin (1992). Fluoxetine: a review of receptor and function effects and their clinical implications. Psychopharmocology 107(1):1-10.

Becnel, J, O. Johnson, J. Luo, D. R. Nassel, C. D. Nichols. (2011). The serotonin 5HT7Dro receptor is expressed in the brain of Drosophila, and is essential for normal courtship and mating. PLoS One 6(6): e20800. doi:10.1371/journal.pone.0020800

Berger, C., S. Renner, K. Luer, G.M. Technau (2007). The commonly used marker ELAV is transiently expressed in neurobblasts and glial cells in the Drosophila 
embryonic CNS. Developmental Dynamics 236(12):3562-3568.

Berger, A. J., D. A. Bayliss, F. Viana (1992). Modulation of neonatal ray hypoglossal motoneuron excitability by serotonin. Neuroscience Letters 143(1-2):164-168.

Berni, J., A. M. Muldal, S. R. Pulver (2010). Using neurogenetics and the warmthgated ion channel TRPA1 to study the neual basis of behavior in Drosophila. Journal of Undergraduate Neuroscience Education 9(1):A5-A14.

Berni, J., S. R. Pulver, L. C. Griffith, M. Bate (2012). Autonomous circuitry for substrate exploration in feely moving Drosophila larvae. Current Biology 22(20):1861-1870.

Blitz, D. M., M. P. Nusbaum. (2011). Neural circuit flexibility in a small sensorimotor system. Current Opinion in Neurobiology: 21(4):544-552.

Bodmer, R., Y. N. Jan (1987). Morphological differentiation of the embryonic peripheral neurons in Drosophila. Roux's archives of developmental biology 196(2):69-77.

Boher, F., R. Godoy-Herrera, F. Bozinovic (2010). The interplay between thermal tolerance and life history is associated with the biogreography of Drosophila species. Evolutionary Ecology Research 12:1-14. 
Borue, X., B. Condron, B. J. Venton (2010). Both synthesis and reuptake are critical for replenishing the releasable serotonin pool in Drosophila. Journal of Neurochemistry 113:188-199.

Boulanger, A., M. Farge, C. Ramanoudjame, K. Wharton, J-M. Dura. Drosophila motor neuron retraction during metamorphosis is mediated by inputs from TGF- $\beta$ /BMP signaling and orphan nuclear receptors. PLOS One 0040255.

Buchanan, G. F., G. B. Richerson (2010). Central serotonin neurons are required for arousal to $\mathrm{CO}_{2}$. PNAS 107(37):16354-16359.

Butt, S. J. B., O. Kiehn (2003). Functional identification of interneurons responsible for left-right coordination of hindlimbs of mammals. Neuron 38: 953-963.

Brustein, E., M. Chong, B. Holmqvist, P. Drapeau. (2003). Serotonin patterns locomotor network activity in the developing zebrafish by modulating quiescent periods. Journal of Neurobiology 57(3): 303-322.

Cattaert, D., S. Birman (2001). Blockade of the central generator of locomotor rhythm by noncompetitive NMDA receptor antagonists in Drosophila larvae. Journal of Neurobiology 48(1):58-73. 
Cazalets, J. R., Y. Sqalli-Houssani, F. Clarac (1992). Activation of the central pattern generators for locomotion by serotonin and excitatory amino acids in neonatal rat. Journal of physiology 455:187-204.

Cheng, L. E., W. Song, L. L. Looger, L. Y. Jan, Y. N. Jan (2010). The role of the TRP channel NompC in Drosophila larval and adult locomotion. Neuron 67:373-380.

Chollet, F., J. Tardy, J-F Albucher, C. Thalamas, E. Berard, C. Lamy, Y. Bejot, S. Deltour, A. Jaillard, P. Niclot, B. Guillon, T. Moulin, P. Marque, J. Pariente, C. Arnaud, I. Loubinoux (2011). Fluoxetine for motor recovery after acute eschaemic stroke (FLAME): a randomised placebo-controlled trial.

Costedio, M. M., N. Hyman, G. M. Mawe (2006). Serotonin and its role in colonic function and in gastrointestinal disorders. Diseases of the Colon and Rectum (2006) 50(3):376-388.

Christopherson, K. S, E. M. Ullian, C. C. Stokes, C. E. Mullowney, J. W. Hell, A. Agah, J. Lawler, D. F. Mosher, P. Bornstein, B. A. Barres (2005). Thrombospondins are astrocyte-secreted proteins that promote CNS synaptogenesis. Cell 120:421-433.

Crisp, S. J., J. F. Evers, M. Bate (2011). Endogenous patterns of activity are required for maturation of a motor network. The Journal of Neuroscience. 31(29):1044510450. 
Dam, M, P. Tonin, A. De Boni, G. Pizzolato, S. Casson, M. Ermani, U. Freo, L. Piron, L. Battistin (1996). Effects of fluoxetine and maprotiline on function recovery in poststroke hemiplegic patients undergoing rehabilitation therapy. Stroke 27:12111214.

Dasari, S., R. L. Cooper. (2004). Modulation of sensory-CNS-motor circuits by serotonin, octopamine, and dopamine in semi-intact Drosophila larva. Neuroscience Research 48(2):221-227.

Daubert, E., B. Condron (2010). Serotonin: a regulator of neuronal morphology and circuitry. TINS 33(9):424-434.

Dhaka, A., V. Viswanath, A. Patapoutian (2006). TRP ion chennels and temperature sensation. Annual Review of Neuroscience, 29:135-161.

Dickerson, P. (2006). Neuromodulation of central pattern generators in invertebrates and vertebrates. Current Opinion in Neurobiology. 16(6):604-614.

Dillon, M. E., G. Wang, P. A. Garrity, R. B. Huey (2011). Review: Thermal preference in Drosophila. Journal of Thermal Biology, 34(3):109-119.

Feraboli-Lehnherr, D., J.-Y. Barthe, D. Orsal (1999). Serotonin-induced activation of 
the network for locomotion in adult spinal rats. Journal of Neuroscience Research 55(1):87-98.

Fox, L. E., D. R. Soll, C-F Wu. (2006). Coordination and modulation of locomotion pattern generators in Drosophila larvae: effects of altered biogenic amine levels by the tyramine $\beta$ hydroxylase mutation. The Journal of Neuroscience 26(5):14861498.

Friesen, W. O. (1994). Reciprocal Inhibition: A mechanism underlying oscillatory animal movements. Neuroscience and biobehavioral reviews, 18(4):547-553.

Friesen, W. O., J. Cang (2001). Sensory and central mechanisms control intersegmental coordination. Current Opinion in Neurobiology. 11(6):678-683.

Gabriel, J. P., R. Mahmood, A. Kyriakatos, I. Soll, G. Hauptmann, R. I. Calabrese, A. El Manira (2009). Serotonergic modulation of locomotion in zebrafish - endogenous release and synaptic mechanisms. The Journal of Neuroscience 29(33):10387-10395.

Gao, X. J., C. J. Potter, D> M. Gohl, M. Silies, A. Y. Katsov, T. R. Clandinin, L. Luo. (2013). Specific kinematics and motor-related neurons for aversive chemotaxis in Drosophila. Current Biology 23(13):1163-1172. 
Gershon, M. D. (1991). Serotonin: its role and receptors in enteric neurotransmission. Advanced Experiments in Medical Biology 294:221-230.

Gershon, M.D., J. Tack (2007). The serotonin signaling system: from basic understanding to drug development for functional GI disorders. Gastroenterology 132(1):397-414.

Gjorgjieva, J., J. Berni, J. F. Evers, S. J. Eglen (2013). Neural circuits for peristaltic wave propagation in crawling Drosophila larvae: analysis and modeling. Frontiers in Computational Neuroscience. doi: 10.3389/fncom.2013.00024

Harris-Warrick, R. M., A. H. Cohen (1985). Serotonin modulates the central pattern generator for locomotion in the isolated lamprey spinal cord. Journal of Experimental Biology 116:27-46.

Harris-Warrick, R. M. (2011). Neuromodulation and flexibility in central pattern generator networks. Current Opinion in Neurobiology. 21(5):685-692.

Hebbar, S., J. J. Fernandes (2010). Glial remodeling during metamorphosis influences the stabilization of motor neuron branches in Drosophila. Developmental Biology $340: 344-354$.

Heckscher, E. S., S. R. Lockery, C.Q. Doe (2012). Characterization of larval crawling at 
the level of organism, segment, and somatic body wall musculature. The Journal of Neuroscience 32(36):12460-12471.

Heckscher, E. S., A. A> Zarin, S. Faumont, M. Q. Clark, L .Manning, A. Fushiki, C. M. Schneider-Mizell, R. D. Fetter, J. W. Truman, M. F. Zwart, M. Landgraf, A. Cardona, S. Lockery, C. Q. Doe. (2015). Even-skipped+ interneurons are core components of a sensorimotor circuit that maintains left-right symmetric muscle contraction amplitude. Neuron In press.

Hoffman, M.S., G. S. Mitchell. (2011). Spinal 5-HT7 receptor activation induces longlasting phrenic motor facilitation. The Journal of Physiology 589(6):1397-1407.

Hughes, C. L., J. Thomas (2007). A sensory feedback circuit coordinates muscle activity in Drosophila. Molecular and Cellular Neuroscience. 35(2):383-96.

Huizinga, J., W. J. E. P. Lammers (2009). Gut peristalsis is governed by a multitude of cooperating mechanisms. American Journal of Physiology: Gastrointestinal Liver Physiology. 296(1): G1-G8.

Hwang, R. Y., L. Zhong, Y. Zu, T. Johnson, F. Zhang, K. Deisseroth, W. Daniel Tracey. Nociceptive neurons protect Drosophila larvae from parasitoid wasps. Current Biology 17(24):2105-2116. 
Itakura, Y, H. Kohsaka, T. Ohyama, M. Zlatic, S. R. Pulver, A. Nose (2015). Identification of inhibitory premotor interneurons activated at a late phase in a motor cycle during Drosophila larval locomotion. PLoS One DOI: 10.1371/journal.pone.0136660

Iwasaki, T. J. Chen, W. O. Friesen (2014). Biological clockwork underlying adaptive rhythmic movements. Proceedings of the National Academy of Sciences. 111(3):978-983.

Kang, K., V. C. Panzano, E. C. Chang, L. Ni, A. M. Dainis, A. M. Jenkins, K. Regna, M. A. T. Muskavitch, P. A. Garrity (2012). Modulation of TRPA1 thermal sensitivity enables sensory discrimination in Drosophila. Nature 481, 76-80.

Kim, J., Y. D. Chung, D-y. Park, S. Choi, D. W. Shin, H. Soh, H. W. Lee, W. Son, J. Wim, CS. Park, M. J. Kernan, C. Kim (2003). A TRPV family ion channel required for hearing in Drosophila. Nature 424:81-84.

Kohsaka, H., S. Okusawa, Y. Itakura, A. Fushiki, A. Nose. (2012). Development of larval motor circuits in Drosophila. Development, Growth and Differentiation. 54, 408-419.

Kohsaka, H., E. Takasu, T. Morimoto, A. Nose (2014). A group of segmental premotor interneurons regulates the speed of axial locomotion in Drosophila larvae. Current 
Biology 24:1-11.

Koon, A.C., J. Ashley, R. Barria, S. DasGupta, R. Brain, S. Waddell, M. H. Alkema, V. Budnik (2011). Autoregulatory and paracrine control of synaptic and behavioral plasticity by octopaminergic signaling. Nature Neuroscience 14: 190-199.

Knudsen, E. I., P. F. Knudsen (1989). Visuomotor adaptation to displacing prisms by adult and baby barn owls. The Journal of Neuroscience 9(9):3297-3305.

Lahiri S, Shen K, Klein M, Tang A, Kane E, . (2011) Two Alternating Motor Programs Drive Navigation in Drosophila Larva. PLoS ONE 6(8): e23180. doi:10.1371/journal.pone.0023180

Lanuza, G. M., S. Gosgnach, A. Pierani, T. M. Jessell, M. Goulding (2004). Genetic identification of spinal interneurons that coordinate left-right locomotor activity necessary for walking movements. Neuron 42:375-386.

Lee, T., L. Luo (2001). Mosaic analysis with a repressible cell marker (MARCM) for Drosophila neural development. TRENDS in Neurosiences 24(5):251-254

Liu, L, O. Yermolaieva, W. A. Johnson, F. M. Abboud, M. J. Welsh (2003). Identification and function of thermosensory neurons in Drosophila larvae. Nature Neuroscience 6:267-273. 
Schoeffter. (2004). Functional expression of the serotonin 5-HT7 receptor in human glioblastoma cell lines. British Journal of Pharmacology 143:404-410.

Marder, E., D. Bucher (2001). Central pattern generators and the control of rhythmic movements. Current Biology. 11(23): R986-996

Marder, E., D. Bucher, D. J. Schulz, A. L. Taylor (2005). Invertebrate central pattern generators moves along. Current Biology 15(7):R685-R699.

McKemy, D.D., W. N. Neuhausser, D. Julius (2002). Identification of a cold receptor reveals a general role for TRP channels in thermosensation. Nature 416: 52-58.

Millhorn, D. E., F. L. Eldridge, R. G. Waldrop (1980). Prolonged stimulation for respiration by endogenous central serotonin. Respiration Physiology 32(3):171188.

Mullins, O., J. T. Hackett, W. O. Friesen (2011). Local-distributed integration by a novel neuron ensudres rapid initiation of animal locomotion. Journal of Neurophysiology 105(1):130-144. 
Ohyama, T., T. Jovanic, G. Denisov, T. C. Dang, D. Hoffmann, R. A. Kerr, M. Zlatic (2013). High-throughput analysis of stimulus-evoked behaviors in Drosophila larva reveals multiple modality-specific escape strategies. PLOS ONE: 10.1371

Okusawa, S., H. Kohsaka, A. Nose (2014). Serotonin and Downstream Leucokinin Neurons Modulate Larval Turning Behavior in Drosophila. The Journal of Neuroscience 34(7):2544-2558.

Ossipov, M. H., G. O. Dussor, F. Porreca (2010). Central modulation of pain. Journal of Clinical Investigation 120(11):3779-3787.

Park, S., R. George, Y. Cai, H. Y. Chang, D. E. Krantz, F. Friggi-Grelin, S. Birman, J. Hirsh (2006). Cell-type-specific limitation on in vivo serotonin storage following ectopic expression of the Drosophila serotonin transporter, dSERT. Journal of Neurobiology, 66(5):452-462.

Peabody, N. C., J. B. Pohl, F> Diao, A. P. Vreede, D. J. Sandstrom, H. Wang, P. K. Zelensky, B. H. White (2009). Characterization of the decision metwork for wing expansion in Drosophila using targeted expression of the TRPM8 channel. The Journal of Neuroscience 29(11):3343-3353.

Pulver, S., S. L. Pashkovski, N. J. Hornstein, P. A. Garrity, L. C. Griffith (2009). Temporal dynamics of neuronal activation by channelrhodopsin-2 and TRPA1 
determine behavioral output in Drosophila larvae. Journal of Neurophysiology 101(6):3075-3088.

Pulver, S. R., T.G. Bayley, A. L. Taylor, J. Berni, M. Bate, B. Hedwig (2015). Imaging fictive locomotor patterns in larval Drosophila. Journal of Neurophysiology 10.1152/jn.00731.2015

Rajacopalan, M., G. Kurian, J. John (1998). Symptom relief with amitriptyline in the Irritable Bowel Syndrome. Journal of Gastroenterology and Hepatology. 13(7):738741.

Ransdell, J. L., S. S. Nair, D. J. Schultz (2012). Excitability in a central pattern generator network stabilizes functional neural network output. The Journal of Neuroscience 32(28):9649:9658.

Rodriguez Moncalvo, V.G., A.R. Campos (2009). Role of serotonergic neurons in the Drosophila larval response to light. BMC Neuroscience 10:66.

Rosenzweig, M., K. M. Brennan, T. D. Tayler, P. I. Phelps, A. Patapoutian, P. A. Garrity (2006). The Drosophila ortholog of vertebrate TRPA1 regulates thermotaxis. Genes and Development, 19:419-424.

Ruat, M., E. Traiffort, Rob Leurs, J. Tardivel-Lacombe, J. Diaz, J-M. Arrang, J-C 
Schwartz (1993). Molecular cloning, characterization, and localization of a high affinity serotonin receptor (5-HT7) activating cAMP formation. Proceedings of the National Academy of Science 90:8547-8551.

Santello, M., C. Cali, P. Bezzi (2012). Gliotransmission and the tripartite synapse. Advanced Experiments in Medical Biology 970:307-331.

Schoofs, A., S. Huckesfeld, P. Schlegel, A. Miroschnikow, M. Peters, M. Zeymer, R. Spiess, A-S. Chiang, M. J. Pankratz (2014). Selection of motor programs for suppressing food intake and inducing locomotion in the Drosophila brain. PLOS Biology DOI: 10.1371

Shen, WL, Y. Kwon, A. A. Ageqbola, J. Luo, C. Montell. (2011). Function of rhodopsin in temperature discrimination in Drosophila. Science 331(6022):1333-6.

Silva, B., N. I. Goles, R. Varas, J. M. Campusano (2014). Serotonin receptors expressed in Drosophila mushroom bodies differentially modulate larval locomotion. PLoS One 9(2):e89641.

Simon, M. A., B. A. Trimmer (2009). Movement encoding by a stretch receptor in the soft-bodied caterpillar, Manduca sexta. The Journal of Experimental Biology 2121021-1031. 
Simon, M. A., S.J. Fusillio, K. Colman, B. A. Trimmer (2010). Motor patterns associated with crawling in a soft-bodied arthropod. The Journal of Experimental Biology. 213: 2303-2309.

Song, W. ,M. Onishi, L. Y. Jan, Y. N. Jan (2007). Peripheral multidendritic sensory neurons are necessary for rhythmic locomotion behavior in Drosophila larvae. PNAS 104(12):5199-5204.

Stent, G. S., W. B. Kristan, W. O. Friesen, C. A. Ort, M. Poon, R. L. Calabrese (1978). Neuronal generation of the leech swimming movement. Science 200(4348):13481357.

Story, G. M., A. M. Peier, A. J. Reeve, S. R. Eid, J. Mosbacher, T. R. Hricik, T. J. Earley, A. C. Hergarden, D. A. Andersson, S. W. Hwang, P. McIntyre, T. Jegla, S. Bevan, A. Patapoutian (2003). ANKTM1, a TRP-like channel expressed in nociceptive neurons, is activated by cold temperatures. Cell 112(6):819-829.

Tamarkin, D. A., R. B. Levine (1996). Synaptic interactions between a muscleassociated proprioceptor and body wall muscle motor neurons in larval and adult Manduca sexta. Journal of Neurophysiology 76(3):1597-1610.

Tastekin, I., Julia, Riedl, V. Schilling-Kurz, A. Gomez-Marin, J. W. Truman, M. Louis (2015). Role of subesophageal zone in sensorimotor control of orientation in 
Drosophila larva. Current Biology 25(11):1448-1460.

Ting, C-Y., S. Gu, S. Guttikonda, T-Y Lin, B. H. White, C-H. Lee (2011). Focusing transgene expression in Drosophila by coupling Gal4 with a novel split-lexA expression system. Genetics 188(1):229-233.

Tracey, W. D., R. I. Wilson, G. Laurent, S. Benzer (2003). painless, a Drosophila gene essential for nociception. Cell 113(2):261-273.

Tsuboushi, A., J. C. Caldwell, W. D. Tracey. (2012). Dendritic filopodia, ripped pocket, NOMPC, and NMDARs contribute to the sense of touch in Drosophila larvae. Current Biology. 22(22):2124-2134.

Valles, A. M., K. White (1988). Serotonin-containing neurons in Drosophila melanogaster: Development and distribution. Journal of Comparative Neurology 268(3):414-428.

Voituron, N., Y. Shvarev, C. Menuet, M. Bevengut, C. Fasano, E. Vigneault, S. El Mestikawy, G. Hillaire (2010). Fluoxetine treatment abolishes the in vitro respiratory response to acidosis in neonatal mice. PLoS One 5(10):e13644.

Weese-Mayer, D. E., E. M. Berry-Kravis, B. S. Maher, J. M. Silvestri, M. E. Curran, M. L. Marazita (2003). Sudden infant death syndrome: Association with a promoter 
polymorphism of the serotonin transporter gene. American Journal of Medical Genetics Part A. 117A(3):268-274.

Weimann, J. M., P. Skiebe, H-G Heinzel, C. Soto, N. Kopell, J. C. Jorge-Rivera, E. Marder (1997). Modulation of oscillator interactions in the crab stomatogastric ganglion by crustacean cardioactive peptide.

Wolfer, D. P., W. E. Crusio, H-P Lipp. (2002). Knockout mice: simple solutions to the problems of genetic background and flanking genes. Trends in Neuroscience 25(7):336-340.

Xiang, Y., Q. Yuan, N. Vogt, L. L. Looger, L. Y. Jan, Y. N. Jan. (2010). Light-avoidancemediating photoreceptors tile the Drosophila larval body wall. Nature 468:921-926.

Yan, Z., W. Zhang, Y. He, D. Gorczyca, Y. Xiange, L. E. Cheng, S. Meltzer, L. Y. Jan, Y. N. Jan (2013). Drosophila NOMPC is a mechanotranscduction channel subunit for gentle-touch sensation. Nature 493:221-225.

Yano, J.M., K. Yu, G. P. Donaldson, G. G. Shastri, P. Ann, L. Ma, C. R. Nagler, R. F. Ismagilov, S. K. Mazmanian, E. Y. Hsaio (2015). Indigenous bacteria from the gut microbiota regulate host serotonin biosynthesis. Cell 161(2):264-76. 